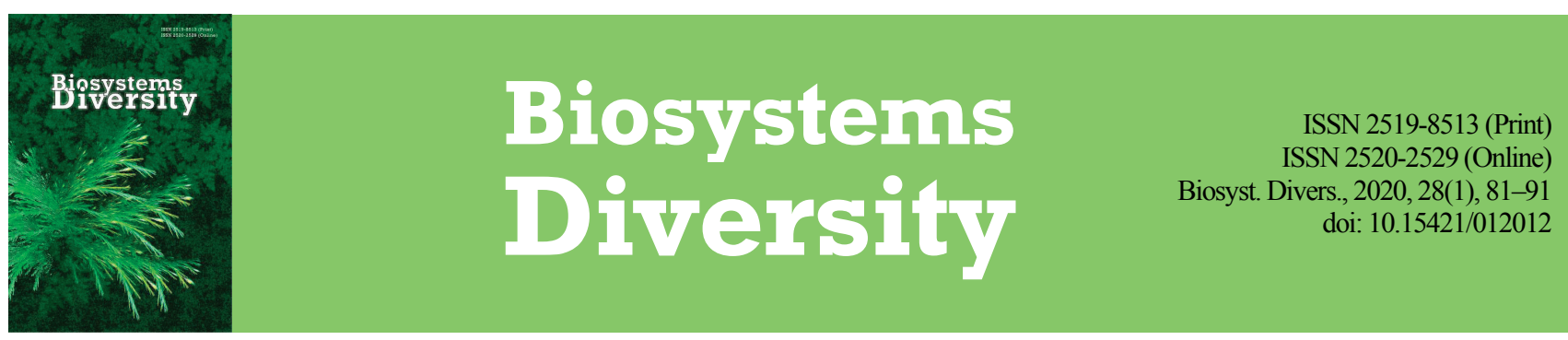

\title{
Floristic diversity of steppe territories near Poltava town (Ukraine)
}

\author{
D. A. Davydov*, L. M. Gomlya** \\ *M. G. Kholodny Institute of Botany of National Academy of Sciences of Ukraine, Kyiv, Ukraine \\ **V. G. Korolenko Poltava National Pedagogical University, Poltava, Ukraine
}

Article info

Received 22.12.2019

Received in revised form 19.01.2020

Accepted 20.01.2020

M. G. Kholodny Institute of Botany, NAS of Ukraine,

Tereshchenkivska st., 2,

Kyiv, 01004, Ukraine.

Tel.: +38-066-982-03-52.

E-mail:tovarystwo@gmail.com

\section{Davydov, D. A., \& Gomlya, L. M. (2020). Floristic diversity of steppe territories near Poltava town (Ukraine). Biosystems Di- versity, 28(1), 81-91. doi:10.15421/012012}

Current progress in botany requires new claims for floristic research. Now the latter is not a simple species inventory of a separate local or regional flora but it needs coordination with recent results of critical taxonomic, nomenclatural and molecular phylogenetic investigations. Based on the fact that detailed research on steppes as a zonal type of vegetation in the ForestSteppe zone of Ukraine is very important for preservation of current steppe territories, the authors studied several territories with steppe vegetation near Poltava town (Poltava region, Ukraine). The key steppe territories found are situated near Abazivka, Rozhayivka, Kostochky, Buhayivka, Machukhy, Ivonchentsi and Zhuky villages. Data about steppe flora from only the first territory located between Abazivka and Rozhayivka villages including "Rozhayivskyi" local botanical reserve were early reported in literature sources while data about steppe vegetation of the other areas has never been published in detail. The full list of 401 vascular plant species found on these steppe territories with the frequency of distribution, major synonym names and references to current taxonomic papers for separate species are proposed. One of these species (Hemerocallis fulva (L.) L.) is a new alien for Poltava region. Taxonomy for all species was critically revised, nomenclature of several taxa (Dichoropetalum carvifolia (Vill.) Pimenov \& Kljuykov, Erophila verna (L.) DC., Campanula canescens (Waldst. \& Kit.) Roth) is discussed in detail. The name "Dichoropetalum carvifolium-chabraei (Crantz) Soldano et al." is an invalid designation based on trinominal and must be rejected. The names Selinum chabraei Jacq. ex Murray, Peucedanum euphimiae Kotov and Hemerocallis lilio-asphodelus var. fulva L. were lectotypified. The studied steppe territories have the great significance in the sozological aspect, they include 32 rare steppe plant species (seven from the Red Data Book of Ukraine and 25 from the list of locally rare plants within Poltava region) so the primary task for further research is to organize their protection as the most valuable steppe areas and the monitoring of their condition in the future.

Keywords: vascular plants; taxonomy; floristic findings; steppes; Poltava region.

\section{Introduction}

The study and conservation of plant diversity is one of the most relevant topics in currect botany. The Shenzhen Declaration adopted at the XIX International Botanical Congress (23-29 July 2017, Shenzhen, China) endorses seven major priorities for strategic actions in the plant sciences. Two of them are to accelerate the inventory of life on Earth for the wise use of nature and the benefit of humankind and to value, document and protect indigenous, traditional and local knowledge about plants and nature (Crane et al., 2017). So detailed floristic research on natural plant communities for their preservation is nowadays very important.

The main aim of floristic research is to provide botanical inventory of separate local or regional flora associated with some territory. This is the one of the simplest forms of botanical study and is rather popular since it needs no special equipment. The final result of this study may be publicshed mostly in three forms: as a checklist (a short vascular plant species list without any comments), a conspectus (a checklist with comments on plant species distribution, ecology, conservation status, etc.) and a flora (a fundamental monographic paper with identification keys, morphological descriptions, detail data about plants species area of distibution, etc.).

Advances in molecular biology and plant taxonomy in the past 30 years require a new approach to regional floristics. The new revolutionary system of angiosperm plant classification elaborated by the Angiosperm Phylogeny Group was published between 1998 (APG, 1998) and 2016 (APG IV, 2016). The nomenclatural data based on results of the Linnean Plant Name Typifications Project (Jarvis, 1992, 2007) and similar studies on the plant name typification (Moberg \& Nilsson, 1991; Al-Shehbaz \& Barriera, 2019; Peruzzi et al., 2019) have great significance for plant taxonomists. Many current authors have emphasized that both mole- cular phylogenetic and nomenclatural data are to be summarized in current floristic checklists, conspectuses and floras (Heywood, 2000; Li, 2008). The one of recent fundamental working projects based on these principles is "Flora of Uzbekistan" (Sennikov et al., 2016) but similar studies have been previously organized and realized for Iran with adjacent areas (Akhani, 2006) and Turkey with the East Aegean Islands (Davis et al., 1998). During the last 10 years several resumptive plant checklists and conspectuses for separate Europaean countries were also published for the Czech Republic (Danihelka et al., 2012), Greece (Dimopoulos et al., 2013), Albania (Barina et al., 2018), Finland (Kurtto et al., 2019). So floristic studies and critical inventories of vascular plants on local and regional levels are relevant and necessary now.

Steppe communities are among the zonal vegetation types of Ukraine but they need conservation since a large number of sites with this vegetation are currently destroyed. Previously steppe areas were quite widespread in the Forest-Steppe zone of Ukraine but now in Poltava region they occupy small areas on ravine and river valley slopes. The flora and vegetation of steppe territories distributed near Poltava town have not been studied completely. Some information existing in literature sources (Illichevskyi, 1927, 1928; Andrienko et al., 1996; Bayrak-Smolyar \& Korotchenko, 1996; Bayrak, 1997; Bayrak \& Stetsiuk, 2005, 2008; Shaparenko, 2012) is fragmentary, partly outdated and mostly associated with separate threatened vascular plant species. During the last 15 years (2005-2019) we studied steppe vegetation in Poltava district and found several valuable steppe plots not mentioned in botanical literature sources. The only wellknown territory presented in this paper is "Rozhayivskyi" local botanical reserve; detailed information about its flora was generalized in our previous paper (Davydov \& Gomlya, 2016). 


\section{Materials and methods}

This research is based on the data of the field work by the authors in 2005-2019 on the steppe territories distributed near Poltava town and in Poltavskyi administrative district of Poltava region (Oblast) in general. For studying the flora of steppes of this region we chose five the most valuable key locations which hold numerous rare species. The first location (AR - Abazivka-Rozhayivka) is situated on slopes of the left bank of the Poluzirya River between Abazivka and Rozhayivka villages. It consists of the territory of "Rozhayivskyi" local botanical reserve and adjacent steppe plots. A list of vascular plants species found by us in this location was published in 2016 (Davydov \& Gomlya, 2016). However one of us (D.A. Davydov) on his field expedition in 2017 and 2018 found here several new species not mentioned in our previous paper so they have been added and indicated in the text as "!" for AR. The second location (K - Kostochky) includes steppe communities on the slopes of the right bank of the Poluzirya River near Kostochky village. The third steppe complex ( $\mathrm{B}$ - Buhayivka) is located on ravine slopes near Buhayivka village. The fourth territory ( $\mathrm{M}$ - Machukhy) has a cluster structure including several ravine complexes distributed to the south of Poltava town near Machukhy village. The fifth territory ( $\mathrm{ZZ}$ - Ivonchentsi-Zhuky) neighbouring upon the borders of Poltava town (Ivonchentsi) is situated on slopes near Zhuky village (Fig. 1).

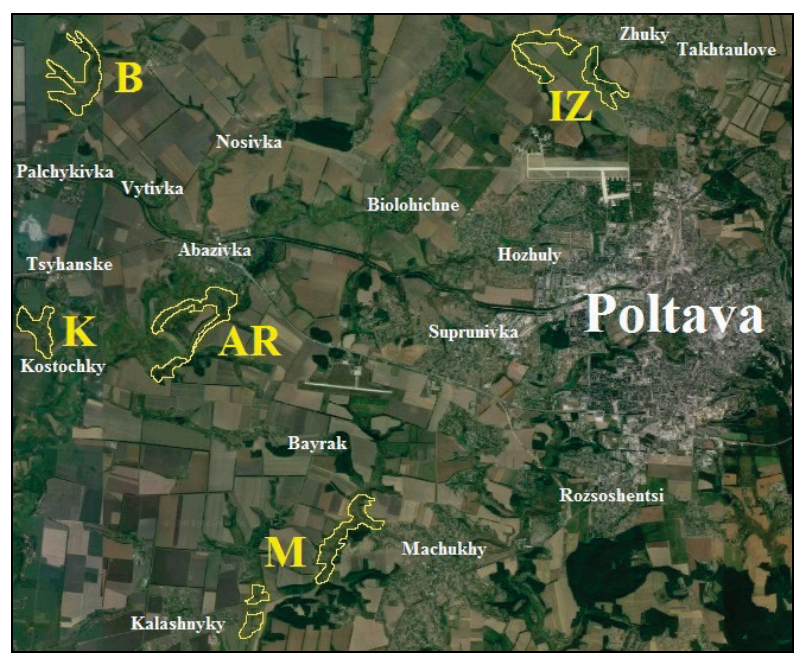

Fig. 1. The arrangement of studied steppe territories on the satellite map by Google Inc.: AR - Abazivka-Rozhayivka, B - Buhayivka,

IZ - Ivonchentsi and Zhuky, K - Kostochky, M-Machukhy

The first step of our research was to find all species of vascular plants on all designated steppe locations. This work was realized by our field expeditions (in 2005-2007 - by both authors, in 2008-2019- by D. A. Davydov). Based on field results, literature sources and the analysis of herbarium specimens from the herbaria of M. G. Kholodny Institute of Botany (KW) and V. G. Korolenko Poltava National Pedagogical University (PWU) we compiled the annotated checklist of vascular plants of steppe territories found near Poltava town. In this checklist the frequency of distribution is presented by the following scale: $\mathrm{c}$ (common) - this species is common on all (or almost all) plots of the studied steppe locations and forms numerous populations; nc (not common) - the species is found on many (but not all) plots of the studied steppe locations and it is not very common and characteristic; $r$ (rare) - the species is found on several plots of studied steppe location, it is locally distributed; $\operatorname{vr}$ (very rare) - the species is very rare on this territory, it was found only several times in different years; "-" - the species has not been found on this territory. Sozological status for the vascular plants found has been indicated before the plant species names according to current nature conservation documents: “***" - the third edition of the Red Data Book of Ukraine (Chervona, 2009); “*”- the list of locally rare plants within Poltava region adopted by Annex 2 to the Decision of the 18th session of the fourth convocation of the Poltava Regional Council in February 2004 (Bayrak \& Stetsiuk, 2005). The second step of our research was critical analysis of taxonomy and nomenclature of vascular plant species found on the studied steppe territories. It has beed made by D. A. Davydov, who analyzed more than 500 publications with original descriptions (protologues) of steppe plant taxa and taxonomic papers about their current systematic positions based on results of both traditional morphological and molecular phylogeny research by different authors. So the main synonym names are indicated in our list with very brief comments (if needed) and citations of major systematic revisions for separate problematic taxa.

Herbarium specimens of steppe plant species from studied locations (about 1000 sheets) are preserved in herbaria of V. G. Korolenko Poltava National Pedagogical University (PWU) and M. G. Kholodny Institute of Botany of NAS of Ukraine (KW).

\section{Results}

The species composition of the analyzed steppe flora includes 401 species from 237 genera and 52 families:

Equisetaceae

1. Equisetum arvense L.: $\mathrm{AR}(\mathrm{r}), \mathrm{K}(\mathrm{nc}), \mathrm{B}(\mathrm{nc}), \mathrm{M}(\mathrm{nc}), \mathrm{IZ}(\mathrm{nc})$.

2. E. ramosissimum Desf.: $\mathrm{K}(\mathrm{nc})$.

Aristolochiaceae

3. Aristolochia clematitis L.: B(r).

Colchicaceae

4. **Colchicum versicolor Ker Gawl. (=Bulbocodium versicolor (Ker Gawl.) Spreng.): AR(r), K(r). On the name of this taxon (Persson, 2007; Mosyakin, 2013).

Liliaceae

5. Gagea erubescens (Besser) Schult. \& Schult. f.: AR(nc), K(nc), B(nc), $\mathrm{M}(\mathrm{nc}), \mathrm{IZ}(\mathrm{nc})$.

6. G. pusilla (F. W. Schmidt) Sweet: AR(nc), K(nc), B(nc), M(c). About the authorship of this species (Bayer \& López González, 1989). Iridaceae

7. **Crocus reticulatus Steven ex Adams: AR(nc), K(c), B(c), M(nc), IZ(r).

8. **Gladiolus imbricatus L. (=G. temuis M. Bieb., G. apterus Klokov): $\mathrm{AR}(\mathrm{vr})$.

9. *Iris aphylla L. (=I. hungarica Waldst. \& Kit.): AR(r), B(nc).

10. *I. pumila L.: AR(r) Asphodelaceae

11. Hemerocallis fulva (L.) L.: B(vr) Alliaceae

12. Allium oleraceum $\mathrm{L} .: \mathrm{AR}(\mathrm{nc}), \mathrm{K}(\mathrm{nc}), \mathrm{B}(\mathrm{nc}), \mathrm{M}(\mathrm{nc}), \mathrm{Z}(\mathrm{nc})$.

13. A. rotundum L. (=A. waldsteinii G. Don f.): $\mathrm{AR}(\mathrm{nc}), \mathrm{K}(\mathrm{nc}), \mathrm{B}(\mathrm{nc})$, $\mathrm{M}(\mathrm{nc}), \mathrm{IZ}(\mathrm{nc})$.

14. A. sphaerocephalon $\mathrm{L}: \mathrm{K}(\mathrm{nc})$. Agavaceae

15. Anthericum ramosum L.: B(r). Hyacinthaceae

16. Asparagus officinalis L. (=A. polyphyllus Steven): AR(nc), K(nc), $\mathrm{B}(\mathrm{nc}), \mathrm{M}(\mathrm{nc}), \mathrm{IZ}(\mathrm{nc})$.

17. *Bellevalia ciliata (Cirillo) T. Nees ( $=$ B. sarmarica Pall. ex Woronow, B. speciosa Woronow ex Grossh.): $\mathrm{K}(\mathrm{vr})$. On this taxon name (Berg et al., 1989).

18. *Hyacinthella leucophaea (K. Koch) Schur: AR(nc), K(r), B(nc), M(r).

19. *Muscari neglectum Guss. ex Ten.: AR(nc), K(vr), B(nc), M(nc), IZ(vr). Cyperaceae

20. Carex hirta L.: AR(c), K(c), B(nc), M(c), IZ(c).

21. C. melanostachya M. Bieb. ex Willd.: K(r), B(nc), M(nc).

22. C. michelii Host: AR(r!), B(r).

23. C. praecox Schreb.: AR(c), K(c), B(c), M(c), IZ(c).

24. C. supina Willd. ex Wahlenb.: AR(r).

25. C. tomentosa $\mathrm{L}: \mathrm{AR}(\mathrm{nc})$.

Poaceae

26. *Aegilops cylindrica Host: B(r).

27. Agropyron cristatum (L.) P. Beauv. s.l. (incl. A. pectinatum (M. Bieb.) P. Beauv., A. stepposum Dubovik): AR(nc), K(nc), B(nc), M(nc), IZ(r).

28. Agrostis vinealis Schreb.: AR(nc), K(nc).

29. Alopecurus pratensis L.: AR(nc), K(nc), B(nc), M(nc), IZ(nc).

30. Arrhenatherum elatius (L.) P. Beauv. ex J. Presl \& C. Presl: AR(nc), $\mathrm{K}(\mathrm{nc}), \mathrm{B}(\mathrm{c}), \mathrm{M}(\mathrm{c}), \mathrm{IZ}(\mathrm{nc})$.

31. Bothriochloa ischaemum (L.) Keng: AR(r!).

32. Bromus arvensis $\mathrm{L} .: \mathrm{AR}(\mathrm{r}), \mathrm{B}(\mathrm{nc})$. 
33. B. hordeaceus L. (=B. mollis L.): AR(r), K(r), B(r), IZ(r). On the correct name of this species (Krasniak, 2012).

34. B. inermis Leyss. (=Bromopsis inermis (Leyss.) Holub): AR(c), K(c), $\mathrm{B}(\mathrm{c}), \mathrm{M}(\mathrm{c}), \mathrm{IZ}(\mathrm{c})$. About the circumscription of the genus Bromus $\mathrm{L}$. (Saarela et al., 2007; Soreng et al., 2017).

35. B. japonicus Thunb.: $\mathrm{B}(\mathrm{nc})$.

36. B. riparius Rehmann (=Bromopsis riparia (Rehmann) Holub): AR(nc), $\mathrm{K}(\mathrm{nc})$.

37. B. squarrosus L.: $\mathrm{AR}(\mathrm{c}), \mathrm{K}(\mathrm{c}), \mathrm{B}(\mathrm{c}), \mathrm{M}(\mathrm{c}), \mathrm{IZ}(\mathrm{nc})$.

38. B. tectorus L. (=Anisantha tectorum (L.) Nevski): AR(nc), K(nc), $\mathrm{B}(\mathrm{nc}), \mathrm{M}(\mathrm{nc}), \mathrm{IZ}(\mathrm{nc})$.

39. Calamagrostis epigejos (L.) Roth: $\mathrm{AR}(\mathrm{c}), \mathrm{K}(\mathrm{c}), \mathrm{B}(\mathrm{c}), \mathrm{M}(\mathrm{c}), \mathrm{IZ}(\mathrm{c})$.

40. Dactylis glomerata L.: AR(nc), K(r), B(nc), M(nc), IZ(c).

41. Echinochloa crus-galli (L.) P. Beauv.: K(vr), B(r), IZ(r).

42. Elymus repens (L.) Gould (=Elytrigia repens (L.) Desv. ex Nevski): $\mathrm{AR}(\mathrm{c}), \mathrm{K}(\mathrm{c}), \mathrm{B}(\mathrm{c}), \mathrm{M}(\mathrm{c}), \mathrm{IZ}(\mathrm{c})$. The generic name Elymus $\mathrm{L}$. is to be accepted against Elytrigia Nevski (Melderis, 1978; Soreng et al., 2017).

43. Festuca valesiaca Gaud. s.l. (incl. F. rupicola Heuff.): AR(c), K(c), $\mathrm{B}(\mathrm{c}), \mathrm{M}(\mathrm{c}), \mathrm{IZ}(\mathrm{c})$.

44. Hierochlöe repens (Host) P. Beauv.: AR(c), K(r), B(nc).

45. Hordeum murinum L. s.l. (incl. H. leporinum Link): IZ(r). Taxonomy (Jacobsen \& Bothmer, 1995).

46. Koeleria macrantha (Ledeb.) Schult. (=K. cristata (L.) Pers. comb. illeg.): AR(c), K(c), B(c), M(c), IZ(nc). On the correct name (Arnow, 1994).

47. Lolium perenne $\mathrm{L}$ : $\mathrm{AR}(\mathrm{nc}), \mathrm{K}(\mathrm{nc}), \mathrm{B}(\mathrm{nc}), \mathrm{M}(\mathrm{r}), \mathrm{ZZ}(\mathrm{r})$.

48. *Melica transsilvanica Schur: AR(r!), K(nc), B(c).

49. Phleum phleoides (L.) H. Karst.: AR(r), K(r).

50. P. pratense L.: AR(nc), K(nc), B(nc), M(nc), IZ(nc).

51. Phragmites australis (Cav.) Trin. ex Steud.: K(nc).

52. Po a апnиa L.: B(r), M(vr).

53. P. bulbosa L.: AR(nc), K(c), B(nc), M(nc), IZ(r).

54. P. compressa L.: AR(r), K(nc), B(nc), M(nc), IZ(r).

55. P. pratensis L. s.l. (incl. P. angustifolia L.): $\mathrm{AR}(\mathrm{c}), \mathrm{K}(\mathrm{c}), \mathrm{B}(\mathrm{c}), \mathrm{M}(\mathrm{c}), \mathrm{ZZ}(\mathrm{c})$.

56. Schedonorus pratensis (Huds.) P. Beauv. (=Festuca pratensis Huds., Lolium pratense (Huds.) Derbysh.): AR(nc), K(nc), B(nc), M(nc), IZ(c). About this genus (Foggi et al., 2005; Tzvelev, 2011).

57. Sclerochloa dura (L.) P. Beauv.: K(vr), B(r), M(r).

58. Setaria pumila (Poir.) Roem. \& Schult. (=S. glauca auct. non (L.) Roem. \& Schult.): AR(r!), K(nc), B(r), M(r), IZ(r). On the correct name (Rauschert, 1973).

59. S. viridis (L.) P. Beauv.: AR(r), K(r), B(r), IZ(r).

60. **Stipa capillata L.: AR(c), K(c), B(c), M(nc), IZ(nc).

61. ${ }^{* *}$ S. pulcherrima K. Koch: AR(r).

62. Thinopyrum intermedium (Host) Barkworth \& D. R. Dewey (=Elytrigia intermedia (Host) Nevski, Elymus hispidus (Opiz) Melderis): $\mathrm{AR}(\mathrm{c}), \mathrm{K}(\mathrm{c}), \mathrm{B}(\mathrm{c}), \mathrm{M}(\mathrm{c}), \mathrm{ZZ}(\mathrm{nc})$. On this genus (Barkworth \& Dewey, 1985; Soreng et al., 2017). Papaveraceae

63. Fumaria schleicheri Soy.-Willem.: AR(vr), B(vr), M(vr), IZ(r). Ranunculaceae

64. **Adonis vernalis L.: AR(nc), K(r), B(nc), M(r).

65. *Anemonoides sylvestris (L.) Galasso et al. (=Anemone sylvestris L.): AR(vr), M(r). On this name (Banfi et al., 2005).

66. Ceratocephala orthoceras DC. $(=$ C. testiculata (Crantz) Besser comb. illeg.): $\mathrm{AR}(\mathrm{r}), \mathrm{K}(\mathrm{r}), \mathrm{B}(\mathrm{nc}), \mathrm{M}(\mathrm{r})$. This name is correct (Gutermann, 2009).

67. *Clematis integrifolia L.: AR(r), K(r), B(r).

68. Delphinium consolida L. (=Consolida regalis $\mathrm{S}$. F. Gray, C. paniculata (Host) Schur): AR(nc), K(r), B(r), M(r), IZ(r). The genus Consoli$d a$ (DC.) S. F. Gray was not accepted (Jabbour \& Renner, 2011; Kadereit et al., 2016).

69. Ficaria verna Huds. s.l. (incl. F. stepporum P. Smirnov): AR(r), K(vr), $\mathrm{M}(\mathrm{r}), \mathrm{IZ}(\mathrm{r})$.

70. Ranunculus acris L.: AR(r), B(r), M(r), IZ(nc).

71. R. illyricus $\mathrm{L}$ : $\mathrm{AR}(\mathrm{nc}), \mathrm{K}(\mathrm{r}), \mathrm{B}(\mathrm{nc})$.

72. R. pedatus Waldst. \& Kit. (=R. silvisteppaceus Dubovik): AR(nc), K(r), IZ(r). Taxonomy (Jelenevskyi \& Derviz-Sokolova, 1989).

73. R. polyanthemos $\mathrm{L}$ : $\mathrm{AR}(\mathrm{c}), \mathrm{K}(\mathrm{nc}), \mathrm{B}(\mathrm{c}), \mathrm{M}(\mathrm{c}), \mathrm{IZ}(\mathrm{c})$.

74. R. repens $\mathrm{L} .: \mathrm{B}(\mathrm{r}), \mathrm{M}(\mathrm{r})$.

75. Thalictrum minus L.: AR(c), K(nc), B(nc), M(c), IZ(nc)

76. T. simplex $\mathrm{L} .: \mathrm{AR}(\mathrm{r} !), \mathrm{B}(\mathrm{r})$.
Fabaceae

77. Anthyllis vulneraria L. s.l. (=A. macrocephala Wender.): $\mathrm{K}(\mathrm{vr}), \mathrm{B}(\mathrm{r})$.

78. Astragalus austriacus Jacq.: AR(nc), K(c).

79. A. cicer $\mathrm{L}$.: $\mathrm{AR}(\mathrm{nc}), \mathrm{K}(\mathrm{nc}), \mathrm{B}(\mathrm{r}), \mathrm{M}(\mathrm{nc}), \mathrm{ZZ}(\mathrm{nc})$.

80. **A. dasyanthus Pall.: AR(r), K(r).

81. A. onobrychis L.: AR(c), K(c), B(c), M(nc), IZ(nc).

82. Caragana arborescens Lam.: AR(vr!).

83. Cytisus austriacus L. (=Chamaecytisus austriacus (L.) Link): AR(c), $\mathrm{K}(\mathrm{c}), \mathrm{B}(\mathrm{nc}), \mathrm{M}(\mathrm{nc}), \mathrm{IZ}(\mathrm{nc})$. The genus Chamaecytisus Link was included in Cytisus L. (Cristofolini \& Troì, 2006; Kadereit et al., 2016).

84. Ervum tetraspermum L. (=Vicia tetrasperma (L.) Schreb.): AR(nc), $\mathrm{K}(\mathrm{nc}), \mathrm{B}(\mathrm{nc}), \mathrm{M}(\mathrm{nc}), \mathrm{ZZ}(\mathrm{nc})$. The genus Ervum L. is to be separated from Vicia L. (Schäfer et al., 2012; Fedoronchuk, 2018).

85. Genista tinctoria L.: AR(c), B(c).

86. *Lathyrus pannonicus (Jacq.) Garcke (=L. lacteus (M. Bieb.) Wissjul.): AR(r), K(r), B(nc), M(r). Taxonomy (Bässler, 1981; Schlee et al., 2011).

87. L. tuberosus L.: AR(nc), K(r), B(nc), IZ(nc).

88. Lotus corniculatus L. s.l. (=L. ucrainicus Klokov, L. stepposus Kramina): AR(nc), K(nc), B(nc), M(nc), IZ(nc).

89. Medicago falcata L. s.l. (incl. M. romanica Prodan): AR(c), K(c), B(c), M(c), IZ(c). Taxonomy (Lesins \& Lesins, 1979).

90. M. lupulina $\mathrm{L}$ : AR(r), K(r), B(nc), IZ(r).

91. M. sativa L.: AR(r), IZ(r).

92. Melilotus albus Medik.: AR(r), B(r), IZ(r).

93. M. officinalis (L.) Pall.: K(r), B(r), IZ(r).

94. Onobrychis arenaria (Kit.) DC. (=O. tanaitica Spreng.): AR(nc), K(r), $\mathrm{B}(\mathrm{r}), \mathrm{M}(\mathrm{nc}), \mathrm{IZ}(\mathrm{nc})$. Taxonomy (Fedoronchuk \& Mosyakin, 2018).

95. * Oxytropis pilosa (L.) DC.: B(r).

96. Robinia pseudoacacia L.: AR(r), K(r), M(vr), IZ(r).

97. Securigera varia (L.) Lassen (=Coronilla varia Lassen): AR(c), K(c), B(c), M(c), IZ(c). Taxonomy (Lassen, 1989; Fedoronchuk \& Mosyakin, 2018).

98. Trifolium alpestre $\mathrm{L} .: \mathrm{AR}(\mathrm{nc}), \mathrm{K}(\mathrm{r})$.

99. T. arvense $\mathrm{L}: \mathrm{AR}(\mathrm{c}), \mathrm{K}(\mathrm{nc}), \mathrm{B}(\mathrm{nc})$.

100. T. medium L.: AR(nc), K(nc), B(c), M(nc).

101. T. montanum L. (=Amoria montana (L.) Soják): AR(c), K(nc), B(c), $\mathrm{M}(\mathrm{c}), \mathrm{IZ}(\mathrm{c})$.

102. T. pratense L.: AR(nc), K(nc), B(nc), M(nc), IZ(c).

103. T. repens L. (=Amoria repens (L.) C. Presl): AR(nc), K(r), B(r), M(r), $\mathrm{IZ}(\mathrm{r})$.

104. Vicia cracca L.: AR(c), K(nc), B(c), M(c), IZ(nc).

105. V. tenuifolia Roth: $\mathrm{AR}(\mathrm{nc}), \mathrm{M}(\mathrm{nc})$.

106. V. villosa Roth: AR(r), K(r), IZ(r).

Polygalaceae

107. Polygala comosa Schkuhr (=P. podolica DC.): AR(nc), K(nc), B(nc). Rosaceae

108. Agrimonia eupatoria L.: AR(c), K(c), B(c), M(c), IZ(nc). Taxonomy (Iamonico, 2017).

109. Argentina anserina (L.) Rydb. (=Potentilla anserina L.): AR(vr), B(r). Taxonomy (Soják, 2010).

110. Crataegus monogyna Jacq. (=C. leiomonogyna Klokov): $\mathrm{K}(\mathrm{r})$, $\mathrm{B}(\mathrm{nc}), \mathrm{M}(\mathrm{r})$. Taxonomy of the genus (Christensen, 1992).

111. C. rhipidophylla Gand. (=C. curvisepala Lindm. nom. illeg.): $\mathrm{AR}(\mathrm{nc}), \mathrm{B}(\mathrm{nc}), \mathrm{M}(\mathrm{r}), \mathrm{IZ}(\mathrm{r})$.

112. Filipendula vulgaris Moench: $\mathrm{AR}(\mathrm{c}), \mathrm{K}(\mathrm{nc}), \mathrm{B}(\mathrm{c})$.

113. Fragaria viridis Weston: AR(c), K(c), B(c), M(c), IZ(c).

114. Geum urbanum L.: AR(r), IZ(r).

115. Malus domestica (Suchow) Borkh.: AR(r), K(r), M(vr), IZ(r).

116. Potentilla argentea L. (=P. impolita Wahlenb., P. neglecta Baumg.): AR(c), K(c), B(c), M(nc), IZ(nc). Taxonomy (Soják, 2004).

117. P. humifusa Willd. ex Schltdl.: AR(nc), K(nc), B(nc), M(nc).

118. P. patula Waldst. \& Kit.: K(r).

119. P. recta L. s.l. (=P. obscura Willd.): AR(c), K(nc), B(nc), M(nc), $\mathrm{IZ}(\mathrm{nc})$.

120. P. reptans L.: $\mathrm{B}(\mathrm{r})$.

121. Prunus armeniaca L. (=Armeniaca vulgaris Lam.): AR(r), K(r), $\mathrm{B}(\mathrm{r}), \mathrm{M}(\mathrm{vr})$. Taxonomy of this genus (Shi et al., 2013).

122. P. cerasus L. (=Cerasus vulgaris Mill.): AR(r).

123. *P. fruticosa Pall. (=Cerasus fruticosa Pall.): K(r), B(vr). 
124. P. spinosa L. (=P. stepposa Kotov): AR(c), K(c), B(c), M(nc), IZ(c).

125. Pyrus communis L. (=P. pyraster (L.) Burgsd.): AR(r), K(r), B(nc), $\mathrm{M}(\mathrm{r}), \mathrm{IZ}(\mathrm{nc})$.

126. Rosa antonovii (Lonacz.) Dubovik: K(vr), IZ(vr).

127. R. borysthenica Chrshan.: M(vr).

128. $R$. canina L. s.l. (incl. $R$. lupulina Dubovik): AR(nc), K(nc), B(nc), $\mathrm{M}(\mathrm{r}), \mathrm{IZ}(\mathrm{r})$

129. *R. chrshanovskii Dubovik (=R. pygmaea M. Bieb. nom. illeg.): K(vr).

130. $R$. corymbifera Borkh.: AR(nc), K(nc), B(nc), M(nc), IZ(nc).

131. R. mediata Dubovik: AR(r), B(r). Taxonomically problematic species, our previous indication on " $R$. caesia Sm." (Davydov \& Gomlya, 2016) belongs here.

132. R. rubiginosa L.: AR(nc), K(nc), M(r), IZ(nc).

133. $R$. sherardii Davies (=R. tomentosa auct. non $\mathrm{Sm}$ ): AR(r), K(r), $\mathrm{M}(\mathrm{r}), \mathrm{IZ}(\mathrm{r})$. On this name (Buzunova, 2000).

134. R. villosa L. (=R. pomifera Herrm.): AR(r!), B(r), M(nc). Taxonomy (Buzunova, 2000).

Elaeagnaceae

135. Elaeagnus angustifolia L.: AR(vr), B(r), M(vr), IZ(vr). Rhamnaceae

136. Rhammus cathartica L.: AR(r), K(r), B(r), M(vr), IZ(vr). Ulmaceae

137. Ulmus minor Mill. (=U. carpinifolia Rupp. ex Suchow, U. suberosa Moench): AR(r!), K(r), B(r), IZ(vr). Taxonomy (Zhygalova, 2016). Urticaceae

138. Urtica dioica L.: AR(nc), K(nc), B(nc), M(nc), IZ(nc). Euphorbiaceae

139. Euphorbia kaleniczenkoi Czern.: AR(r), K(vr), B(nc).

140. E. seguieriana Neck: AR(nc!), K(nc).

141. E. semivillosa Prokh.: AR(nc), K(r), B(nc), M(r).

142. E. stepposa Zoz ex Prokh.: AR(c), K(c), B(c).

143. E. virgata Waldst. \& Kit: AR(c), K(c), B(nc), M(nc), IZ(nc). Violaceae

144. Viola accrescens Klokov: AR(vr), K(r).

145. $V$. ambigua Waldst. \& Kit.: AR(c), K(nc), B(c), M(nc), IZ(nc).

146. $V$. arvensis Murray: AR(nc), B(nc), IZ(r).

147. V. tricolor L. (=V. matutina Klokov): AR(nc), K(r), B(nc), M(nc), IZ(r). Linaceae

148. Linum flavum L.: M(r).

149. L. hirsutum L.: AR(nc).

150. L. nervosum Waldst. \& Kit:: AR(r).

151. *L. perenne L.: AR(vr, cited only by literature source (Shaparenko, 2012)).

Celastraceae

152. Euonymus europaeus L.: AR(vr!). Hypericaceae

153. Hypericum elegans Steph. ex Willd: AR(nc), K(r), B(nc), M(nc), IZ(r).

154. H. perforatum L.: AR(c), K(c), B(c), M(c), IZ(c). Sapindaceae

155. Acer negundo L.: AR(r), K(r), B(r), M(vr), IZ(vr).

156. A. tataricum L.: AR(r), K(r), B(nc), M(r). Malvaceae

157. Malva thuringiaca (L.) Vis. (=Lavatera thuringiaca L.): AR(c), K(nc), $\mathrm{B}(\mathrm{nc}), \mathrm{M}(\mathrm{nc}), \mathrm{IZ}(\mathrm{nc})$. On the current state of the genus Lavatera L. (Banfi et al., 2005; Kadereit et al., 2016). Resedaceae

158. Reseda lutea $\mathrm{L}: \mathrm{AR}(\mathrm{nc}), \mathrm{B}(\mathrm{r}), \mathrm{M}(\mathrm{r})$ Brassicaceae

159. Alyssum alyssoides (L.) L. (=A. calycinum L.): $\mathrm{B}(\mathrm{r})$. This name is correct (German, 2003).

160. A. desertorum Stapf: AR(nc), K(nc), B(nc).

161. Arabidopsis thatiana (L.) Heynh.: K(r), M(r).

162. Berteroa incana (L.) DC.: AR(nc), K(nc), B(nc), M(nc), IZ(nc).

163. Brassica campestris L.: M(vr), IZ(vr).

164. Bunias orientalis L.: AR(nc), K(nc), B(nc), M(nc), IZ(nc).

165. Camelina microcarpa Andrz. (=C. sylvestris Wallr.): $\mathrm{AR}(\mathrm{nc}), \mathrm{K}(\mathrm{nc})$, B(nc), M(nc), IZ(nc). Taxonomy (Smejkal, 1971).

166. Capsella bursa-pastoris (L.) Medik.: AR(nc), K(r), B(nc), M(r), IZ(r).

167. Chorispora tenella (Pall.) DC.: AR(r), K(vr), B(r), M(vr).

168. Descurainia sophia (L.) Webb ex Prantl: AR(nc), B(nc), M(r), IZ(r).
169. Draba nemorosa L.: AR(nc), K(r), B(r), IZ(r).

170. Erophila verna (L.) DC.: AR(nc), K(nc), B(nc), M(r), IZ(nc). Nomenclatural citation see below.

171. Erysimum diffusum Ehrh. (=E. canescens Roth): AR(c), K(nc), B(c), $\mathrm{M}(\mathrm{c}), \mathrm{IZ}(\mathrm{nc})$.

172. E. marschallianum Andrz. ex M. Bieb. (=E. strictum P. Gaertn. et al., E. hieraciifolium L. nom. ambig.): AR(nc), K(r), M(r), IZ(r). Taxonomy (Polatschek, 2010; German, 2015).

173. Euclidium syriacum (L.) R. Br.: M(vr).

174. Guenthera persica (Boiss. \& Hohen.) German (=Erucastrum armoracioides (Czern. ex Turcz.) Cruchet): AR(vr), B(vr), M(r). About this genus (Gómez-Campo, 2002; German, 2015).

175. Hesperis tristis L. (=Hesperidium triste (L.) G. Beck comb. inval., Sperihedium triste (L.) Dorof.): AR(vr!), B(r), M(r).

176. Lepidium densiflorum Schrad.: B(r).

177. L. draba L. (=Cardaria draba (L.) Desv.): K(r), M(nc). On the state of the genus Cardaria Desv. (Al-Shehbaz et al., 2002).

178. Microthlaspi perfoliatum (L.) F. K. Meyer (=Thlaspi perfoliatum L.): AR(nc), K(nc), B(nc). About this genus (Meyer, 1973; Ali et al., 2016).

179. Sinapis arvensis L.: AR(r), B(r), M(r), IZ(vr).

180. Sisymbrium loeselii L.: AR(nc), K(nc), B(nc), M(nc), IZ(nc).

181. S. polymorphum (Murray) Roth: AR(nc), K(nc), B(nc).

182. S. volgense M. Bieb. ex Fourn.: IZ(vr). Specific epithet was corrected (German, 2014).

183. Thlaspi arvense L:: AR(r), K(nc), B(r), M(r), IZ(r).

184. Turritis glabra L.: B(r). Santalaceae

185. Thesium ramosum Hayne (=T. arvense Horvat. nom. illeg.): AR(nc), $\mathrm{K}(\mathrm{nc}), \mathrm{B}(\mathrm{nc}), \mathrm{M}(\mathrm{nc}), \mathrm{IZ}(\mathrm{nc})$. Widely used in many sources, the name T. arvense is illegitimate (Gutermann, 2009).

Viscaceae

186. Viscum album L.: AR(r), M(r), IZ(r). Mostly grows on Crataegus spp. Plumbaginaceae

187. Limonium tomentellum (Boiss.) Kuntze s.l. (=L. alutaceum (Steven) Kuntze): K(nc). Taxonomy (Moysiyenko, 2008).

Polygonaceae

188. Polygonum arenastrum Boreau (=P. aviculare auct. non L.): AR(nc), K(r), B(nc), M(r), IZ(r). Taxonomy (Styles, 1962).

189. P. patulum M. Bieb.: K(nc).

190. Rumex acetosella L.: K(r).

191. R. confertus Willd: K(r), B(r), M(r).

192. R. crispus L.: AR(r), B(r), M(r), IZ(r).

193. R. thyrsiflorus Fingerh.: B(vr), IZ(vr). Caryophyllaceae

194. Alsine media L. (=Stellaria media (L.) DC.): AR(r), M(r), IZ(r).

195. Arenaria serpyllifolia L. (=A. viscida Lois., A. uralensis Pall. ex Spreng.): AR(nc), K(nc), B(nc), M(nc), IZ(nc). Taxonomy (Lazkov, 2006).

196. Cerastium fontanum Baumg. s.l. (=C. holosteoides Fr.): AR(nc), $\mathrm{K}(\mathrm{nc}), \mathrm{B}(\mathrm{nc}), \mathrm{M}(\mathrm{nc}), \mathrm{IZ}(\mathrm{nc})$.

197. Dianthus armeria L.: M(vr).

198. D. campestris M. Bieb. (=D. pseudoversicolor Klokov): K(r).

199. *D. eugeniae Kleopow: IZ(r).

200. Eremogone micradenia (P. Smirnov) Ikonn.: AR(vr), K(r).

201. Gypsophila paniculata L.: AR(r), K(nc), B(nc), M(nc), IZ(nc).

202. Holosteum umbellatum L.: AR(nc), K(nc), B(c), M(nc).

203. Psammophiliella muralis (L.) Ikonn.: AR(nc), K(nc), B(nc), M(nc), IZ(nc).

204. Saponaria officinalis L.: B(r).

205. Scleranthus annuus L.: B(r).

206. Silene chersonensis Zapał. (=Otites chersonensis (Zapał.) Klokov): $\mathrm{AR}(\mathrm{nc}), \mathrm{K}(\mathrm{nc}), \mathrm{B}(\mathrm{nc}), \mathrm{M}(\mathrm{nc}), \mathrm{IZ}(\mathrm{nc})$. Taxonomy of the species (Wrigley, 1986). About the circumscription of Silene L. s.l. (HernándezLedesma et al., 2015).

207. S. chlorantha (Willd.) Ehrh.: AR(r).

208. S. dichotoma Ehrh.: AR(vr), K(vr).

209. S. latifolia Poir. (=Melandrium album (Mill.) Garcke): AR(nc), K(nc), B(nc), M(nc), IZ(nc). Taxonomy (Oxelman et al., 2001; HernándezLedesma et al., 2015).

210. S. nutans L:: AR(nc), B(nc), M(nc), IZ(nc). 
211. S. viscosa (L.) Pers. (=Elisanthe viscosa (L.) Rupr.): AR(r), B(nc), M(r). Taxonomy (Oxelman et al., 2001; Hernández-Ledesma et al., 2015).

212. S. vulgaris (Moench) Garcke (=Oberna behen (L.) Ikonn.): AR(r), M(r). On Silene L. s.l. (Oxelman et al., 2001; Hernández-Ledesma et al., 2015).

213. Stellaria graminea $\mathrm{L} .: \mathrm{AR}(\mathrm{c}), \mathrm{K}(\mathrm{nc}), \mathrm{B}(\mathrm{c}), \mathrm{M}(\mathrm{nc}), \mathrm{Z}(\mathrm{c})$. Amaranthaceae

214. Amaranthus retroflexus L.: AR(r), K(r), B(r), M(r), IZ(r).

215. Polycnemum majus A. Braun ex Bogenh.: M(vr). Nomenclature (Freitag \& Iamonico, 2015).

Chenopodiaceae

216. Atriplex micrantha C. A. Mey.: AR(vr), B(r), M(vr).

217. A. oblongifolia Waldst. \& Kit:: $\mathrm{B}(\mathrm{vr})$, IZ(vr).

218. A. sagittata Borkh. (=A. nitens Schkuhr): AR(vr!), B(r), IZ(r). On the correct name (Suchorukow, 2007).

219. A. tatarica L.: AR(r), K(r), B(r), M(r), IZ(r).

220. Bassia prostrata (L.) Beck (=Kochia prostrata (L.) Schrad.): K(nc). Taxonomy (Hernández-Ledesma et al., 2015; Kadereit et al., 2016).

221. Chenopodiastrum hybridum (L.) S. Fuentes et al. (=Chenopodium hybridum L.): B(vr), IZ(vr). About this genus (Fuentes-Bazan et al., 2012; Kadereit et al., 2016).

222. Chenopodium album L.: AR(r), K(nc), B(nc), M(r), IZ(nc).

223. C. betaceum Andrz. (=C. strictum auct. non Kit.): AR(r), B(r), IZ(vr). Taxonomy (Mosyakin, 2017a).

224. Salsola tragus L. (=S. ruthenica Iljin): M(r). Taxonomy (Mosyakin, 2017b).

Cornaceae

225. Cornus sanguinea L. (=Swida sanguinea (L.) Opiz): AR(r), K(r), B(r), M(r), IZ(r). Taxonomy (Eyde, 1987). Primulaceae

226. Androsace elongata L.: AR(nc), K(nc), B(c), M(nc), IZ(nc).

227. Lysimachia nummularia L.: AR(r), K(nc), B(r), M(r). Rubiaceae

228. Asperula cynanchica L.: AR(c), K(c), B(c), M(nc), IZ(nc).

229. Galium aparine L.: $\mathrm{AR}(\mathrm{r}), \mathrm{K}(\mathrm{nc}), \mathrm{B}(\mathrm{nc}), \mathrm{M}(\mathrm{nc}), \mathrm{ZZ}(\mathrm{nc})$.

230. G. mollugo L. (= G. album Mill.): AR(r), IZ(nc).

231. G. octonarium (Klokov) Soó: AR(c), K(c), B(nc), M(r).

232. G. verum L. (=G. ruthenicum Willd.): AR(c), K(c), B(c), M(c), IZ(c). Apocynaceae

233. *Vinca herbacea Waldst. \& Kit:: M(r).

234. Vincetoxicum hirundinaria Medik. s.l. (=V. albovianum (Kusn.) Pobed., V. stepposum (Pobed.) Á. Löve \& D. Löve): AR(nc), K(nc), $\mathrm{B}(\mathrm{nc}), \mathrm{IZ}(\mathrm{r})$. Boraginaceae

235. Asperugo procumbens L.: AR(vr), K(r), M(vr), $\mathrm{IZ}(\mathrm{vr})$.

236. Buglossoides arvensis (L.) Johnst: $\mathrm{AR}(\mathrm{nc}), \mathrm{K}(\mathrm{nc}), \mathrm{B}(\mathrm{nc}), \mathrm{M}(\mathrm{nc}), \mathrm{ZZ}(\mathrm{nc})$.

237. Cynoglossum officinale L.: AR(nc), K(c), B(nc), M(nc), IZ(nc).

238. Echium vulgare L.: AR(nc), K(nc), B(nc), M(r), IZ(nc).

239. Lappula squarrosa (Retz.) Dumort: AR(nc), K(c), B(nc), M(nc), IZ(nc).

240. Lithospermum officinale $\mathrm{L}$.: AR(r), K(nc), M(r).

241. Lycopsis arvensis L. (=L. orientalis L.): $\mathrm{K}(\mathrm{vr}), \mathrm{M}(\mathrm{vr})$.

242. Myosotis arvensis (L.) Hill: B(r).

243. Nonea rossica Steven: AR(c), K(nc), B(nc), M(nc), IZ(nc). Convolvulaceae

244. Convolvulus arvensis L.: $\mathrm{AR}(\mathrm{c}), \mathrm{K}(\mathrm{c}), \mathrm{B}(\mathrm{c}), \mathrm{M}(\mathrm{c}), \mathrm{IZ}(\mathrm{c})$.

245. Cuscuta approximata Bab. (=C. cupulata Engelm.): AR(vr).

246. C. australis R. Br. s.l. (=C. cesattiana Bertol.): AR(r), K(r), B(r), $\mathrm{M}(\mathrm{r}), \mathrm{ZZ}(\mathrm{r})$.

247. C. epithymum (L.) L.: AR(r), M(nc).

248. C. planiflora Ten.: AR(vr). Solanaceae

249. Datura stramonium L.: B(vr).

250. Hyoscyamus niger L.: AR(r), K(r), B(r), M(r), IZ(vr).

251. Lycium barbarum $\mathrm{L}: \mathrm{K}(\mathrm{nc}), \mathrm{ZZ}(\mathrm{nc})$. Plantaginaceae

252. Linaria biebersteinii Besser s.l. (=L. maeotica Klokov): AR(r), $\mathrm{K}(\mathrm{nc}), \mathrm{B}(\mathrm{nc}), \mathrm{M}(\mathrm{nc}), \mathrm{IZ}(\mathrm{r})$. Taxonomy (Pesskova, 2004).

253. L. vulgaris Mill.: AR(nc), K(nc), B(nc), M(nc), IZ(nc).

254. Plantago lanceolata L.: AR(nc), K(nc), B(nc), M(nc), IZ(c).

255. P. major L.: AR(nc), K(r), B(r), M(r), IZ(r).
256. P. media L. (=P. urvillei Opiz, P. stepposa Kupr.): AR(c), K(c), B(c), M(c), IZ(c). Taxonomy (Shipunov, 2000).

257. Veronica arvensis $\mathrm{L} .: \mathrm{K}(\mathrm{nc}), \mathrm{B}(\mathrm{nc})$.

258. V. austriaca L. (=V. dentata F. W. Schmidt): AR(r), K(r), B(nc), M(r).

259. $V$. chamaedrys L. (=V. vindobonensis (M.A. Fisch.) M.A. Fisch.): AR(c), K(nc), B(c), M(c), IZ(c). Taxonomy (Bardy et al., 2010).

260. V.jacquinii Baumg.: AR(c), K(nc), B(nc), M(nc).

261. V. incana L.: IZ(r).

262. V. polita Fr:: AR(nc), K(nc), B(r).

263. V. prostrata L.: AR(nc), K(c), B(c), M(c), IZ(nc).

264. V. spicata L. s.1. (=V. barrelieri Schott, V. steppacea Kotov): AR(c), $\mathrm{K}(\mathrm{c}), \mathrm{B}(\mathrm{nc}), \mathrm{M}(\mathrm{nc})$. Taxonomy (Bardy et al., 2011).

265. V. spuria $\mathrm{L} .: \mathrm{B}(\mathrm{vr})$.

266. V. teucrium $\mathrm{L}: \mathrm{AR}(\mathrm{r}), \mathrm{B}(\mathrm{r}), \mathrm{M}(\mathrm{r})$.

267. V. verna $\mathrm{L}: \mathrm{AR}(\mathrm{r})$. Scrophulariaceae

268. Verbascum chaixii Vill. s.l. (=V. orientale M. Bieb. nom. illeg., V. marschallianum Ivanina \& Tzvelev): AR(nc), K(nc), B(nc), M(nc), IZ(r).

269. V. lychnitis L.: AR(c), K(c), B(c), M(c), IZ(nc).

270. V. phlomoides L.: AR(r), M(r), IZ(nc).

271. V. phoeniceum L.: AR(nc), K(nc), B(nc). Lamiaceae

272. Ajuga chia (L.) Schreb. (=A. glabra C. Presl, A. pseudochia Des.Shost.): AR(nc), K(r), B(nc), M(nc).

273. A. genevensis L.: AR(nc), $\mathrm{K}(\mathrm{nc}), \mathrm{B}(\mathrm{nc}), \mathrm{IZ}(\mathrm{nc})$.

274. Ballota nigra L.: AR(r), K(r), M(r), IZ(r).

275. Betonica officinalis L.: AR(nc), K(c), B(c), M(nc), IZ(nc).

276. Clinopodium vulgare L.: AR(r), M(r).

277. Glechoma hederacea L.: AR(c), K(nc), B(nc), M(nc), IZ(nc).

278. Lamium amplexicaule L. (=L. paczoskianum Worosch.): AR(nc), $\mathrm{K}(\mathrm{nc}), \mathrm{B}(\mathrm{r}), \mathrm{M}(\mathrm{r}), \mathrm{ZZ}(\mathrm{nc})$. Taxonomy (Mennema, 1989).

279. L. purpureum L.: $\mathrm{B}(\mathrm{r})$.

280. Leonurus villosus Desf. ex D'Urv. (=L. quinquelobatus Gilib. nom. inval.): B(vr), IZ(vr). Nomenclature (Holub, 1993).

281. Nepeta pannonica L.: B(r).

282. Origanum vulgare L.: AR(nc), K(nc), B(nc), IZ(nc).

283. Phlomis pungens Willd.: AR(nc).

284. Phlomoides tuberosa (L.) Moench (=Phlomis tuberosa L.): AR(nc), $\mathrm{K}(\mathrm{nc}), \mathrm{B}(\mathrm{nc}), \mathrm{M}(\mathrm{nc})$. About this genus (Kamelin \& Machmedov, 1990; Mathiesen et al., 2011; Mosyakin, 2013).

285. Prunella vulgaris L.: AR(r), K(nc), B(r), M(r), IZ(r).

286. Salvia nemorosa L. (=S. tesquicola Klokov \& Pobed., S. illuminata Klokov): AR(c), K(c), B(c), M(c), IZ(c).

287. S. nutans L.: AR(c), K(c), B(c).

288. S. pratensis L.: $\mathrm{AR}(\mathrm{nc}), \mathrm{B}(\mathrm{nc})$.

289. S. verticillata L.: AR(c), K(c), B(nc), M(c), IZ(c).

290. Stachys annua (L.) L. (=S. neglecta Klokov nom. inval.): AR(r), $\mathrm{K}(\mathrm{r}), \mathrm{B}(\mathrm{r}), \mathrm{M}(\mathrm{r}), \mathrm{ZZ}(\mathrm{r})$.

291. S. recta L. (=S. transsilvanica Schur): AR(c), K(c), B(c), M(c), IZ(c). Taxonomy (Chrtek, 1992).

292. Teucrium chamaedrys L.: K(nc), M(nc).

293. Thymus marschallianus Willd.: AR(c), K(c), B(c), M(c), IZ(c).

294. Ziziphora acinos (L.) Melnikov (=Acinos arvensis (Lam.) Dandy, Clinopodium acinos (L.) Kuntze): AR(nc), K(nc), B(nc), M(nc). Current status of the genus Acinos Mill. and its taxonomy (Brauchler et al., 2010; Kadereit et al., 2016; Melnikov, 2016). Orobanchaceae

295. Euphrasia pectinata Ten.: AR(r).

296. Odontites vulgaris Moench: AR(nc), K(nc), B(nc), M(nc), IZ(nc).

297. Orobanche alba Steph. ex Willd.: AR(r), K(r), B(nc). Campanulaceae

298. Campanula bononiensis L.: AR(nc), K(nc), B(nc), M(nc).

299. *C. canescens (Waldst. \& Kit.) Roth (=Asyneuma canescens (Waldst. \& Kit.) Griseb. \& Schenk): AR(nc), K(r), M(r), IZ(vr). Nomenclatural citation see below.

300. C. glomerata L. (incl. C. farinosa Andrz): AR(nc), K(r), M(nc), IZ(r).

301. *C. persicifolia L.: AR(r), B(r), M(vr).

302. C. rapunculoides L.: AR(nc), K(r), M(r), IZ(r).

303. C. sibirica L:: AR(nc), K(nc), B(nc), M(r), IZ(nc). Asteraceae 
304. Achillea millefolium L. s.1.: AR(c), K(c), B(c), M(c), IZ(c). This species was accepted in very broad sense including $A$. pannonica Scheele, A. setacea Waldst. \& Kit., A. collina J. Becker ex Rchb. and A. stepposa Klokov \& Krytzka.

305. A. nobilis L.: AR(nc), K(nc), M(nc).

306. Ambrosia artemisiifolia L.: AR(nc), K(nc), B(nc), M(r), IZ(nc).

307. Arctium tomentosum Mill.: AR(r), K(r), B(r), M(r), IZ(r).

308. Artemisia absintium L.: AR(c), K(c), B(c), M(c), IZ(c).

309. A. austriaca Jacq.: AR(c), K(c), B(c), M(c), IZ(c).

310. A. pontica L.: K(nc).

311. A. santonicum L.: K(nc).

312. A. scoparia Waldst. \& Kit.: AR(r), M(r).

313. A. vulgaris L.: AR(c), K(c), B(c), M(c), IZ(c).

314. *Aster bessarabicus Bernh. ex Rchb. (=A. amelloides Besser nom. illeg.): AR(r), K(vr).

315. Carduus acanthoides L.: AR(nc), K(c), B(nc), M(nc), IZ(nc).

316. C. hamulosus Ehrh. (=C. pseudocollinus (Schmalh.) Klokov): B(r). Taxonomy (Kazmi, 1964).

317. C. nutans L. s.l. (=C. thoermeri Weinm., C. attenuatus Klokov): $\mathrm{AR}(\mathrm{vr}), \mathrm{M}(\mathrm{vr})$.

318. Carlina biebersteinii Bernh. ex Hornem.: B(r), M(r).

319. Centaurea diffusa Lam.: AR(nc), K(nc), B(nc), M(c), IZ(nc).

320. C. jacea L. (=C. substituta Czerep.): AR(c), K(nc), B(c), M(c), IZ(c).

321. * ${ }^{*}$. orientalis L.: AR(r), M(r).

322. C. scabiosa L. s.l. (incl. C. apiculata Ledeb., C. adpressa Ledeb., C. pseudocoriacea Dobrocz.): AR(c), K(c), B(c), M(c), IZ(c). Taxonomy (Mikheev, 2000).

323. C. stoebe L. s.l. (incl. C. biebersteinii DC., C. pseudomaculosa Dobrocz.): AR(nc), K(nc), B(r), M(nc), IZ(nc). Lectotypification of this Linnaean name (Greuter, 2003b).

324. Cichorium intybus L.: AR(c), K(c), B(c), M(nc), IZ(c).

325. Cirsium setosum (Willd.) Besser: AR(nc), K(nc), B(nc), M(r), IZ(nc).

326. C. ukranicum Besser ex DC.: AR(nc), B(nc), M(nc), IZ(nc).

327. C. vulgare (Savi) Ten.: AR(nc), K(nc), B(r), IZ(c).

328. *Cota tinctoria (L.) J. Gay (=Anthemis tinctoria L., A. subtinctoria Dobrocz.): AR(r), K(r), B(c), M(r), IZ(r). About this genus (Greuter et al., 2003; Oberprieler et al., 2007; Sonboli et al., 2012; Kadereit et al., 2016).

329. Crepis foetida L. s.l. (incl. C. rhoeadifolia M. Bieb., Barkhausia rhoeadifolia (M. Bieb.) M. Bieb.): AR(nc), K(nc), B(nc), M(nc), IZ(nc). Taxonomy (Greuter, 2003c).

330. C. tectorum L.: K(r), B(nc), IZ(r).

331. Echinops sphaerocephalus L.: AR(r), K(r), B(nc), IZ(r).

332. Erigeron acris L.: B(vr).

333. E. annuus (L.) Pers. (=Phalacroloma anumm (L.) Dumort., Stenactis annua (L.) Nees): AR(nc), K(nc), B(c), M(nc), IZ(nc). Taxonomy (Greuter, 2003a; Sennikov \& Kurtto, 2019).

334. E. canadensis L. (=Conyza canadensis (L.) Cronq.): B(r), IZ(r). Taxonomy (Greuter, 2003a; Kadereit et al., 2016).

335. E. podolicus Besser: AR(nc), K(nc), B(r), IZ(r).

336. *Galatella villosa (L.) Rchb. f. (=Crinitaria villosa (L.) Cass., Linosyris villosa (L) DC.): AR(r!), K(nc). Taxonomy (Greuter, 2003a; Kadereit et al., 2016).

337. Helianthus annuus L.: AR(vr!).

338. Helichrysum arenarium (L.) Moench: AR(nc), K(nc), B(nc), M(nc).

339. Hieracium robustum Fr.: AR(vr).

340. H. umbellatum L.: AR (r).

341. H. virosum Pall.: AR(nc), K(nc), B(nc), M(nc), IZ(nc).

342. Iva xanthiifolia Nutt. (=Cyclachaena xanthiifolia Fresen.): AR(r!), $\mathrm{K}(\mathrm{r}), \mathrm{B}(\mathrm{r}), \mathrm{M}(\mathrm{r}), \mathrm{IZ}(\mathrm{vr})$. Taxonomy and nomenclature (Greuter, 2003d; Pruski, 2005).

343. Jacobaea doria (L.) G. Gaertn. et al. (=J. schwetzowii (Korsh.) Tatanov \& Vasjukov, Senecio schwetsowii Korsh.): M(vr). Taxonomy (Calvo \& Aedo, 2015).

344. J. erucifolia (L.) G. Gaertn. et al. (=Senecio erucifolius L.): B(r), M(r), IZ(r). About the genus Jacobaea Mill. (Pelser et al., 2006; Kadereit et al., 2016).

345. J. racemosa (M. Bieb.) Pelser s.l. (=Senecio paucifolius S. G. Gmel.): AR(vr), M(vr). Taxonomy (Calvo et al., 2013, 2014).
346. J. vulgaris Gaertn. (=Senecio jacobaea L.): AR(nc), K(nc), B(nc), $\mathrm{M}(\mathrm{nc}), \mathrm{IZ}(\mathrm{nc})$.

347. Jurinea arachnoidea Bunge: AR(nc), K(nc), B(nc), M(r).

348. *J. multiflora (L.) B. Fedtsch.: K(vr).

349. Lactuca serriola L.: AR(nc), K(nc), B(nc), M(nc), IZ(nc).

350. L. saligna L.: AR(r), K(vr), B(nc), M(r).

351. L. tatarica (L.) C.A. Mey.: AR(vr), M(vr), IZ(vr)

352. Leucanthemum vulgare (Vaill.) Lam. s.l. (incl. L. ircutianum DC.): $\mathrm{AR}(\mathrm{r}), \mathrm{K}(\mathrm{r}), \mathrm{B}(\mathrm{r})$.

353. Onopordum acanthium L.: AR(r), K(nc), B(r), M(nc), IZ(r).

354. Pentanema britannicum (L.) D. Gut. Larr. et al. (=Inula britannica L.): $\mathrm{AR}(\mathrm{r}), \mathrm{K}(\mathrm{r}), \mathrm{B}(\mathrm{r}), \mathrm{M}(\mathrm{r}), \mathrm{IZ}(\mathrm{r})$. On the recircumscription of the genus Pentanema Cass. (Boiko et al., 2018; Gutiérrez-Larruscain et al., 2018).

355. P. ensifolium (L.) D. Gut. Larr. et al. (=Inula ensifolia L.): AR(vr).

356. $P$. salicinum (L.) D. Gut. Larr. et al. s.l. (incl. P. asperum (Poir.) G. V. Boiko \& Korniy.): AR(nc), K(nc), B(nc), M(nc).

357. Picris hieracioides L:: AR(c), K(nc), B(nc), M(c), IZ(c).

358. Pilosella bauhinii (Besser) Arv.-Touv. aggr. (=Hieracium bauhinii Besser, H. hispidissimum Rehmann, H. plicatulum (Zahn) Juxip): AR(nc), K(nc), B(nc), M(r), IZ(nc).

359. P. brachiata (Lam.) F. Schultz \& Sch. Bip. (=Hieracium brachiatum (Lam.) Naeg. \& Peter): AR(r), B(vr).

360. P. echioides (Lumn.) F. Schultz \& Sch. Bip. (=Hieracium echioides Lumn.): AR(nc), B(r), M(r)

361. P. officinarum F. Schultz \& Sch. Bip. (=Hieracium pilosella L.): AR(c), K(c), B(c), M(c), IZ(c).

362. P. praealta (Vill. ex Gochn.) F. Schultz \& Sch. Bip. (=Hieracium praealtum Vill. ex Gochn., H. piloselloides auct. non Vill.): AR(nc), $\mathrm{B}(\mathrm{nc}), \mathrm{M}(\mathrm{nc}), \mathrm{IZ}(\mathrm{nc})$.

363. Podospermum canum C. A. Mey. (=Scorzonera laciniata auct. non L., S. cana (C. A. Mey.) Hoffm.): K(nc), M(r).

364. Scorzoneroides autumnalis (L.) Moench (=Leontodon autumnalis L.): AR(nc), K(r), B(r), M(r), IZ(nc). About this genus (Greuter et al., 2006; Kadereit et al., 2016)

365. Senecio vernalis Waldst. \& Kit: AR(r), B(r), IZ(r).

366. Solidago canadensis L.: K(vr), IZ(vr).

367. S. virgaurea L.: IZ(r)

368. Sonchus arvensis L.: AR(r), K(r), M(r), IZ(r).

369. S. oleraceus L.: AR(vr), B(vr), M(vr), IZ(vr)

370. Tanacetum corymbosum (L.) Sch. Bip. (=Pyrethrum corymbosum (L.) Scop.): B(vr). Taxonomy (Greuter et al., 2003; Oberprieler et al., 2007; Sonboli et al., 2012).

371. T. vulgare L.: AR(c), K(c), B(c), M(c), IZ(c).

372. Taraxacum officinale Wigg. aggr.: AR(nc), K(c), B(c), M(c), IZ(c).

373. T. serotinum (Waldst. \& Kit.) Poir:: AR(nc), K(nc), B(nc), M(nc), $\mathrm{IZ}(\mathrm{nc})$.

374. Tragopogon dubius Scop. s.l. (incl. T. major Jacq.): AR(nc), K(nc), $\mathrm{B}(\mathrm{nc}), \mathrm{M}(\mathrm{nc}), \mathrm{IZ}(\mathrm{nc})$.

375. T. orientalis L.: B(vr), M(nc), IZ(nc).

376. Tripleurospermum inodorum (L.) Sch. Bip. (=Matricaria perforata Merat): AR(r), K(nc), B(r), M(r), IZ(r)

377. Tussilago farfara L.: AR(r), K(r), B(r), M(nc), IZ(nc).

378. Xanthium albinum (Widder) H. Scholz: M(vr)

379. X. strumarium L.: B(vr). Sambucaceae

380. Sambucus nigra L.: AR(r), K(r), B(vr), M(r), IZ(vr). Caprifoliaceae

381. Cephalaria uralensis (Murr.) Schrad. ex Roem. \& Schult.: AR(nc), $\mathrm{K}(\mathrm{nc}), \mathrm{IZ}(\mathrm{r})$.

382. Knautia arvensis (L.) Coult.: AR(c), K(nc), B(nc), M(nc), IZ(nc).

383. Scabiosa ochroleuca L:: AR(c), K(c), B(c), M(nc), IZ(c).

384. *Valeriana officinalis L. s.l.: AR(vr!), K(r), B(vr) Apiaceae

385. Anthriscus sylvestris (L.) Hoffm.: AR(c), K(nc), B(c), M(nc), IZ(nc).

386. Carum carvi L.: M(r).

387. Chaerophyllum bulbosum L. (=C. prescottii DC.): AR(vr), B(r), IZ(nc).

388. Conium maculatum L:: AR(r), K(vr), M(vr), IZ(r).

389. Daucus carota L.: AR(c), K(nc), B(nc), M(nc), IZ(nc).

390. Dichoropetalum carvifolium (Vill.) Pimenov \& Kljuykov (=Peucedanum carvifolium Vill.): AR(r), M(r). See nomenclatural citation below. 
391. Eryngium campestre L.: AR(c), K(c), B(c), M(c), IZ(c).

392. Falcaria vulgaris Bernh.: AR(c), K(c), B(c), M(c), IZ(c).

393. Heracleum sibiricum L.: AR(nc), K(r), B(nc), M(nc), IZ(nc).

394. Pastinaca sativa L. (=P. sylvestris Mill.): K(nc), IZ(nc). Taxonomy (Pimenov \& Ostroumova, 2012).

395. Pimpinella saxifraga L.: AR(nc), K(nc), B(nc), M(nc), IZ(c).

396. Seseli annum L.: AR(r), B(r), IZ(nc).

397. S. tortuosum L. (=S. campestre Besser): AR(nc), K(nc), B(nc), M(nc), IZ(nc). Taxonomy (Pimenov \& Ostroumova, 2012).

398. Silaum silaus (L.) Schinz \& Thell.: K(r). Taxonomy (Pimenov \& Ostroumova, 2012).

399. Torilis japonica (Houtt) DC.: AR(r), K(r), B(r), M(r), IZ(r).

400. *Trinia kitaibelii M. Bieb.: AR(vr, cited only by literature source (Bayrak \& Stetsiuk, 2005)).

401. Xanthoselinum alsaticum (L.) Schur (=Peucedanum lubimenkoanum Kotov): AR(nc), K(nc), B(nc), M(nc). Taxonomy (Pimenov \& Ostroumova, 2012).

\section{Discussion}

Nomenclature of several accepted species names is to be discussed here in detail.

Dichoropetalum carvifolia (Vill.) Pimenov \& Kljuykov, 2007, Willdenowia, 37(2): 478. - Pencedanum carvifolia Vill. 1779, Prosp. Hist. Pl. Dauphiné: 25; Vinogradova, 2004, Fl. Eur. Orient. 11: 394. Holandrea carvifolia (Vill.) Reduron et al. 1997, J. Bot. Soc. Bot. France, 1: 93. Lectotype (Frey, 1989): [icon] Crantz, 1767, Stirp. Austr. Fasc. 3, tab. 3, fig. 2.

= Selinum chabraei Jacq. ex Murray, 1784, Syst. Veg., ed. 14: 279. Pencedanum chabraei (Murray) Rchb. ex Moessler, 1827, Handb. Gewachsk. ed. 2, 1: 448. Lectotype (designated here by D. A. Davydov): [icon] Jacq. 1773, Fl. Austr. 1: 46, tab. 72.

= Selinum podolicum Besser, 1809, Prim. Fl. Galiciae Austriac. 2: 392. - Peucedanum podolicum (Besser) Eichw. 1830, Skizze: 155; Schischkin, 1951, Fl. URSS, 17: 195; Kotov, 1955, Fl. RSSU, 7: 591. Lectotype (Fedoronchuk, 2007): "Selinum podolicum. Prim. Fl. Gal. Herb. W. Besser" (KW).

= Pencedanum euphimiae Kotov, 1940, Bot. Zhurn. AN URSR, 1(2): 278; id. 1955, Fl. RSSU, 7: 593. Lectotype (designated here by D. A. Davydov; Shiyan, 10.VIII.2015, as "holotype"): "Russian Federation, Rostov region, Latonovo village, on the right bank of Sarmatska bottom. 18.VIII.1923. Y. M. Lavrenko" (KW 115634). Holotype of this species collected by Y. M. Lavrenko in Sarmatska bottom in 1924 was preserved in Kharkiv Agricultural Institute (Kotov, 1940) but it has been probably destroyed. However, the specimen from this location (locus classicus) collected by Lavrenko in 1923 (one year earlier) with the label verified by M. I. Kotov 09.01 .1938 is present in KW, so it is the best choice for the lectotypification procedure.

Incorrect designations: "Peucedanum carvifolium-chabrael" Soldano, 2005, Annot. Checkl. Italian Vasc. Fl.: 20, nom. inval. - "Holandrea carvifolium-chabraei" Soldano et al. 2005, Atti Soc. Ital. Sci. Nat. Mus. Civico Storia Nat. Milano, 146(2): 232, nom. inval. - "Dichoropetalum carvifolium-chabrael" Soldano et al. 2011, Atti Soc. Ital. Sci. Nat. Mus. Civico Storia Nat. Milano, 152(2): 89; Kljuykov et al., 2015, Atti Soc. Ital. Sci. Nat. Mus. Civico Storia Nat. Milano, 2(2): 133, nom. inval.

The genus Dichoropetalum Fenzl is to be separated from Peucedanum L. based on recent molecular phylogenetic studies (Pimenov et al., 2007; Pimenov \& Ostroumova, 2012; Kadereit et al., 2016). Italian botanists (Conti et al., 2005; Banfi et al., 2011) adopted the epithet "carvifoliumchabrael" instead of "carvifolia", believed that two words firstly used by Crantz and accepted later by Jacquin and Allioni form a valid epithet and should be hyphenated. But one of us (D. A. Davydov) has no doubts that the names based on epithet "carvifolium-chabrael" are incorrect (Greuter, 2009; Verloove \& Lambinon, 2014). The names "Selinum carvifolium Chaebraei" and "Selinum carvifolium Gmelini" adopted by Crantz in 1766 and several later authors are trinominals and the phrase "carvifolium Chaebrael" consists of two words not intended as a specific epithet in according to Art. 23.6(b) of International Code of Nomenclature for Algae, Fungi, and Plants (Shenzhen Code; Turland et al., 2018). So the first validly published name for this species is Peucedanum carvifolium Vill.
Based on our results the authorship of the name Erophila verna (Brassicaceae) needs correction:

Erophila verna (L.) DC. IV 1821, Mém. Mus. Hist. Nat. 7: 249; Besser, 1822, Enum. Pl. Volhyn.: 71; Chevall, 1827, Fl. Gen. Env. Paris, ed. 2, 2: 898; N. Busch, 1939, Fl. URSS, 8: 456 (cum. auct. Bess.); Kotov, 1953, Fl. RSSU, 5: 355 (cum. auct. Bess.); id. 1979, Fl. Part. Eur. URSS, 4: 120 (cum. auct. Bess.); Iljinska, 2007, Ecofl. Ukr.: 68; Dorofeyev, 2012, Consp. Fl. Eur. Orient. 1: 407. - E. vulgaris DC. V 1821, Syst. Nat. 2: 356. Lectotype (Jafri, 1973): “Herb. Linn. No. 823.7”(LINN).

We think that the correct name for the taxon previously khown as Asyneuma canescens (Campanulaceae) is Campanula canescens.

Campanula canescens (Waldst. \& Kit.) Roth, 1827, Enum. Pl. Phaen. Germ. 1: 716. - Phyteuma canescens Waldst. \& Kit. 1799-1802, Descr. Icon. Pl. Hung. 1: 12, t. 14. - Asyneuma canescens Griseb. \& Schenk, 1852, Arch. Naturgesch. (Berlin), 18: 335; An. Fedorov, 1957, Fl. URSS, 24: 411; Wissjulina, 1961, Fl. RSSU, 10: 446; An. Fedorov, 1978, Fl. Part. Eur. URSS, 3: 236.

Current phylogenetic studies of Campanulaceae demonstrated that the genus Campanula L. is not monophyletic in traditional circumscription and the species of this genus fall into at least four major clades containing other genera of the family (Kadereit et al., 2016). We think that the better taxonomic solution in this case is to accept Campanula in broad sense with inclusion in the latter of all species from the genera Adenophora Fisch., Asyneuma Griseb. \& Schenk, Legousia Durand and Phyteuma L.

Our results show that floristic research gives interesting data for futher critical taxonomic analysis of the taxa found. We pointed out that names of 91 plant species accepted by the last Ukrainian nomenclatural checklist (Mosyakin \& Fedoronchuk, 1999) were changed. The major part of them belongs to circumscription changing of separate species taxa (mostly inclusion in others) - 40 species (Allium rotundum and A. waldsteinii, Tragopogon dubius and T. major, Seseli tortuosum and $S$. campestre, etc.) and to transferring to different genera mostly based on new molecular phylogenetic data - 43 species (Pyrethrum corymbosum and Tanacetum corymbosum, Colchicum versicolor and Bulbocodium versicolor, Chenopodium hybridum and Chenopodiastrum hybridum, etc.). Five species names previously accepted by S. L. Mosyakin and M. M. Fedoronchuk included typographic or other errors which needed correction: Calamagrostis epigejos (instead of incorrectly cited "C. epigeios"), Euphorbia seguieriana (against "E. seguierana"), Sisymbrium volgense (against "S. wolgensis"), Artemisia santonicum (instead of "A. santonica"), Cirsium ukranicum (against "C. ukrainicum"). The authorship of three plant names was clarified: Erophila verna (L.) DC. (instead of E. verna (L.) Besser, see above), Gagea pusilla (F. W. Schmidt) Sweet (instead of G. pusilla (F. W. Schmidt) Schult. \& Schult. f.) and Polycnemum majus A. Braun ex Bogenh. (against P. majus A. Braun nom. inval.).

The total number of vascular plant species on the studied key steppe territories: Abazivka-Rozhayivka - 319 species (79.6\%), Kostochky 278 (69.3\%), Buhayivka - 289 (72.1\%), Machukhy - 257 (64.1\%), Ivonchentsi-Zhuky -230 species (57.4\%).

We found that 158 vascular plant species $(39.4 \%)$ occur on all five studied territories. The major part of them are very common dominant or characteristic plants for Ukrainian steppe communities (Festuca valesiaca, Bromus inermis, Elymus repens, Ranunculus polyanthemos, Galium verum, etc.). 54 species were found on four key steppe territories (13.5\% - Gagea pusilla, Galium octonarium, Helichrysum arenarium, Adonis vernalis, etc.), 58 - on three territories (14.5\% - Carex melanostachya, Reseda lutea, Viola arvensis, Rumex confertus, etc.), 57 - on two territories (14.2\% - Iris aphylla, Bromus arvensis, Silene vulgaris, Carduus nutans, etc.). 74 species (18.5\%) are of great interest, they were found only on the one territory: Abazivka-Rozhayivka 23 species (5.7\%; Bothriochloa ischaemum, Linum hirsutum, Euonymus europaeus, Hieracium umbellatum, Helianthus annuus, etc.), Buhayivka - 21 (5.2\%; Hemerocallis fulva, Aegilops cylindrica, Turritis glabra, Veronica spuria, Carduus hamulosus, etc.), Kostochky - 15 (3.7\%; Equisetum ramosissimum, Bellevalia ciliata, Bassia prostrata, Jurinea multiflora, etc.), Machukhy - 10 (2.5\%; Linum flavum, Vinca herbacea, Salsola tragus, Xanthium albinum, Carum carvi, etc.), Ivonchentsi-Zhuky - 5 (1.2\%; Hordeum murinum, Sisymbrium volgense, Dianthus engeniae, Veronica incana, Solidago virgaurea). 
The full species list of vascular plant species published here has great significance for regional floristic studies. It includes 13 species not mentioned in a recent publication for Poltava administrative region (Bayrak \& Stetsiuk, 2008). Nine of them (Malus domestica, Prumus armeniaca, P. cerasus, Rosa mediata, Linum hirsutum, Cuscuta planiflora, Crepis foetida, Hieracium robustum and Pilosella brachiata), however, have been earlier reported by us from Abazivka-Rozhayivka location (Davydov \& Gomlya, 2016); one (Rosa antonovii) - indicated by us for Poltava district (Gomlya \& Davydov, 2008), one (Helianthus annuus as a casual alien) cited by Dvirna (2012), one (Rosa borysthenica) - cited by Dubovik (1989) and only the one species (Hemerocallis fulva) has never been mentioned for Poltava region previously.

Hemerocallis fulva (L.) L. 1762, Sp. Pl., ed. 2: 462; Czerniakowska, 1935, Fl. URSS, 4: 56; Bordzilowski, 1950, Fl. RSSU, 3: 87; Ikonnikov, 1979, Fl. Part. Eur. URSS, 4: 217. - H. lilio-asphodelus var. fulva L. 1753 , Sp. Pl.: 324. Lectotype (designated here by D. A. Davydov): "Herb. Linn. No. 446.2"'(LINN).

This ornamental plant from Eastern Asia which is widely distributed in Ukraine sometimes occurs in many regions as an escaped but not naturalized (as casual alien ergaziophyte or relict of cultivation) species. One of us (D. A. Davydov) found it in these locations in Poltava region: 1) Chutove district, near Iskrivka village, border of the forest plantation between "Iskrivka" and "Skorokhodove" railway stations, 24.07.2011, 06.05.2014; 2) Poltava town (Vakulentsi), a meadow area on sands on the left bank of the Vorskla River, 26.05.2013; 3) Mashivka district, Mashivka urban-type settlement, border of the forest plantation near the railroad Poltava-Lozova, 08.07.2013; 4) Novi Sanzhary district, Prystantsiyne village, border of the alder forest near the railroad, 22.04.2014; 5) Poltava district, near Buhayivka village, on steppe slope above a pond, 24.06.2014; 6) Poltava district, Mynivka village, escaped from cultivations near "Mynivka" railway platform, 03.07.2014; 7) Karlivka district, between Fedorivka and Nyzhnia Lanna villages, near the railway bridge on the Lanna River, 08.07.2014; 8) Poltava district, near Kliushnyky village, in oak-pine forest, 27.03.2015; 9) Reshetylivka district, Shkurupiyi village, near "Shkutupiyi" railway platform, 07.05.2015, 07.07.2016; 10) Lubny town, near railroads, 10.06.2018; 11) Lubny district, Vyly village, escaped from cultivation near "Vyly" railway station, 10.06.2018; 12) Kremenchuk district, between Bondari and Bazaluky villages, border of the forest plantation near "Bilany" railway platform. Also this species was found as an alien in 2014-2019 in many locations of Kharkiv (Kolomak, Nova Vodolaha and Kehychivka districts), Kyiv (Boryspil, Brovary and Pereyaslav-Khmelnytskyi districts) and Sumy (Bilopillia district) regions within the Dnipro River Left-Bank area.

Key steppe territories found by us near Poltava town have great significance as outposts of the steppe vegetation. They hold 32 rare steppe plant species. Seven of them are included in the Red Data Book of Ukraine and have national conservation status and 25 from the list of locally rare plants within Poltava region and are regionally rare. Several of them are shown on Figure 2.

The Abazivka-Rozhayivka steppe location comprises all seven nationally and 17 regionally rare species. Kostochky - five nationally and 14 regionally rare species, Buhayivka - three nationally and 12 regionally rare species, Machukhy - three nationally and nine regionally rare species, Ivonchentsi-Zhuky - two nationally and four regionally rare species.



Fig. 2. Several rare plant species found on key steppe territories near Poltava town: A - Stipa capillata L. as dominant on slopes between Abazivka and Rozhayivka villages (17.07.2018); B - Astragalus dasyanthus Pall. in "Rozhayivskyi” local botanical reserve (17.07.2018); C-Stipa pulcherrima

K. Koch on slope near the road between Abazivka and Rozhayivka villages (09.06.2018); D - Adonis vernalis L. near Buhayivka village (06.05.2015); E-Crocus reticulatus Steven ex Adams near Buhayivka village (07.04.2010); F-Muscari neglectum Guss. ex Ten. between Umantsivka and Buhayivka villages (06.05.2015), G-Colchicum versicolor Ker Gawl. in "Rozhayivskyi” local botanical reserve (05.04.2010); H - Iris aphylla L. near Buhayivka village (06.05.2015), I - Hyacinthella leucophaea (K. Koch) Schur between Umantsivka and Buhayivka villages (06.05.2015), J-Prunus fruticosa Pall. near Kostochky village (20.07.2018). All photographs by D. A. Davydov

The most widely distributed rare species are Crocus reticulatus, Stipa capillata (Red Data Book of Ukraine), Muscari neglectum and Cota tinctoria (regionally rare) found on all studied territories. Otherwise, 12 rare species (Gladiolus imbricatus, Stipa pulcherrima, Iris pumila, Bellevalia ciliata, Aegilops cylindrica, Oxytropis pilosa, Rosa chrshanovskii, Dianthus eugeniae, Vinca herbacea and Jurinea multiflora, including two not confirmed by our data Linum perenne and Trinia kitaibelii) were found only the on one steppe territory and belong to the very locally distributed category. Monitoring of their state through further investigations is necessary.

The studied key steppe territories near Poltava town are highly representative, they cover populations of 32 rare steppe species from 33 found in Poltava district (except Pedicularis kaufmannii Pinzger occuring on slopes between Vytivka and Abazivka villages) and from 68 (47.1\%) found in steppes of Poltava region (Davydov \& Gomlya, unpublished data). So the preservation of these locations as objects of the Ukrainian nature reserve fund is a priority objective.

\section{Conclusion}

Our study of the vascular plants on key steppe territories near Poltava emphasized their important scientific value as plots with high plant diversity for the steppe sites within the Forest-Steppe zone of Ukraine. The primary task for further research is to organize the protection of these most 
important steppe areas and the monitoring for their condition in the future. In our opinion, regional floristic studies are very promising and relevant today. However, in the light of the current development of botany they need be coordinated and connected with new data about taxonomy and nomenclature of separate taxa (species typification, critical revision of their circumscription based on new molecular phylogenetic data, discussion on the phylogeny and evolution of high rank taxa, etc.). With the supporting of such conditions the obtained results will have not only local but global scientific significance. Sometimes the study of new papers in the field of taxonomy or nomenclature with the new names presented there instead of the widely accepted ones causes their categorical rejection. However, it is unacceptable to willfully ignore these publications.

The authors are grateful to Valeria Pavlenko-Barysheva and Anna Bashtovenko (Hordiychuk) for their technical assistance in field work near Abazivka village in 2010 and 2014 respectively. This paper is partly based on the research provided by the grant support of the Ukrainian State Fund for Fundamental Research F-83/53427.

\section{References}

Akhani, H. (2006). Flora Iranica: Facts and figures and a list of publications by K. H. Rechinger of Iran and adjacent areas. Rostaniha, 7(suppl. 2), 19-61.

Ali, T., Schmuker, A., Runge, F., Solovyeva, I., Nigrelli, L., Paule, J., Buch, A.K., Xia, X., Ploch, S., Orren, O., Kummer, V., Linde-Laursen, I., Ørgaard, M., Hauser, T. P., Çelik, A., \& Thines, M. (2016). Morphology, phylogeny, and taxonomy of Microthlaspi (Brassicaceae: Coluteocarpeae) and related genera. Taxon, 65(1), 79-98.

Al-Shehbaz, I. A., \& Barriera, G. (2019). Typification of Edmond Boissier's Cruciferae (Brassicaceae) names enumerated in Flora Orientalis. Boissiera, $72,1-192$.

Al-Shehbaz, I. A., Mummenhoff, K., \& Appel, O. (2002). Cardaria, Coronopus, and Stroganowia are united with Lepidium (Brassicaceae). Novon, 12(1), 5-11.

Andrienko, T. L., Bayrak, O. M., Zaludiak, M. I., Kliestov, M. L., Lytvynenko, V. G., Samorodov, V. M., \& Stetsiuk, N. O. (1996). Zapovidna krasa Poltavshchyny [Reserved beauty of Poltava region]. Astreya, Poltava.

APG (1998). An ordinal classification for the families of flowering plants. Annals of the Missouri Botanical Garden, 85(4), 531-553.

APG IV (2016). An update of the Angiosperm Phylogeny Group classification for the orders and families of flowering plants: APG IV. Botanical Journal of the Linnean Society, 181(1), 1-20.

Arnow, L. A. (1994). Koeleria macrantha and K. pyramidata (Poaceae): Nomenclatural problems and biological distinctions. Systematic Botany, 19(1), 6-20.

Banfi, E., Galasso, G., \& Soldano, A. (2005). Notes on systematics and taxonomy for the Italian vascular flora. 1 . Atti della Società Italiana di Scienze Naturali e del Museo Civico di Storia Naturale di Milano, 146(2), 219-244.

Bardy, K. E., Albach, D. C., Schneeweiss, G. M., Fischer, M. A., \& Schönswetter, P. (2010). Disentangling phylogeography, polyploid evolution and taxonomy of a woodland herb (Veronica chamaedrys group, Plantaginaceae s.1.) in Southeastern Europe. Molecular Phylogenetics and Evolution, 57(2), 771-786.

Bardy, K. E., Schönswetter, P., Schneeweiss, G. M., Fischer, M. A., \& Albach, D. C. (2011). Extensive gene flow blurs species boundaries among Veronica barrelieri, V. orchidea and V. spicata (Plantaginaceae) in Southeastern Europe. Taxon, 60(1), 108-121.

Barina, Z., Somogyi, G., Pifko, D., \& Rakaj, M. (2018). Checklist of vascular plants of Albania. Phytotaxa, 378(1), 1-339.

Barkworth, M. E., \& Dewey, D. R. (1985). Genomically based genera in the perennial Triticeae of North America: Identification and membership. American Journal of Botany, 72(5), 767-776.

Bässler, M. (1981). Revision von Lathyrus L. sect. Lathyrostylis (Griseb.) Bässler (Fabaceae). Feddes Repertorium, 92(3), 179-254.

Bayer, E., \& López González, G. (1989). Nomenclatural notes on some names in Gagea Salisb. (Liliaceae). Taxon, 38(4), 643-645.

Bayrak, O. M. (1997). Konspekt flory Livoberezhnoho Prydniprovya. Sudynni roslyny [Conspectus of the flora of the Left Bank of Dnipro River Area. Vascular plants]. Verstka, Poltava (in Ukrainian).

Bayrak, O. M., \& Stetsiuk, N. O. (2005). Atlas ridkisnykh i znykayuchykh roslyn Poltavshchyny [Atlas of rare and endangered plants of Poltava region]. Verstka, Poltava (in Ukrainian).

Bayrak, O. M., \& Stetsiuk, N. O. (2008). Konspekt flory Poltavshchyny. Vyshchi sudynni roslyny [Conspectus of the flora of Poltava region. Higher vascular plants]. Verstka, Poltava (in Ukrainian).

Bayrak-Smolyar, O. M., \& Korotchenko, I. A. (1996). Florystychna i tsenotychna kharakterystyka stepiv Poltavshchyny [Floristic and coenotic characteristics of steppes of Poltava region]. Ukrainian Botanical Journal, 53(5), 554-561 (in Ukrainian).
Berg, C., Knapp, H. D., Messner, U., \& Wiehle, W. (1989). Bellevalia ciliata (Cyr.) Nees (Hyacinthaceae) neu für Bulgarien. Folia Geobotanica et Phytotaxonomica, 24(3), 297-304

Boiko, G. V., Korniyenko, O. M., \& Mosyakin, S. L. (2018). New nomenclatural combinations for taxa of Pentanema (Asteraceae) occurring in Ukraine. Ukrainian Botanical Journal, 75(5), 436-440.

Buzunova, I. O. (2000). Vidy roda Rosa L. (Rosaceae) sektsii Caninae DC. podsektsii Vestitae Christ vo flore Vostochnoy Yevropy i Kavkaza [Species from the genus Rosa L. (Rosaceae) section Caninae DC. subsection Vestitae Christ in the flora of the Eastern Europe and the Caucasus]. Novitates Systematicae Plantarum Vascularium, 32, 61-72 (in Russian).

Calvo, J., \& Aedo, C. (2015). A taxonomic revision of the Eurasian/Northwestern African Senecio doria group (Compositae). Systematic Botany, 40(3), 900-913.

Calvo, J., Álvarez, I., \& Aedo, C. (2014). Three new combinations and a replacement name in Eurasian Senecio (Compositae, Senecioneae). Novon, 23(2), $139-142$.

Calvo, J., Álvarez, I., Aedo, C., \& Pelser, P. B. (2013). A phylogenetic analysis and new delimitation of Senecio sect. Crociseris (Compositae: Senecioneae), with evidence of intergeneric hybridization. Taxon, 62(1), 127-140.

Christensen, K. I. (1992). Revision of Crataegus sect. Crataegus and nothosect. Crataeguineae (Rosaceae-Maloideae) in the Old World. Systematic Botany Monographs, 35, 1-199.

Chrtek, J. (1992). Taxonomical remarks on the Stachys recta group. Folia Geobotanica et Phytotaxonomica, 27(2), 177-183.

Conti, F., Abbate, G., Alessandrini, A., \& Blasi, C. (2005). An annotated checklist of the Italian vascular flora. Palombi Editori, Roma.

Crane, P. R., Ge, S., Hong, D.-Y., Huang, H.-W., Jiao, G.-L., Knapp, S., Kress, W. J., Mooney, H., Raven, P. H., Wen, J., Wu, W.-H., Yang, H.-M., Zhu, W.-H., \& Zhu, Y.-X. (2017). The Shenzhen Declaration on Plant Sciences - Uniting plant sciences and society to build a green, sustainable Earth. PhytoKeys, 86, 3-7.

Cristofolini, G., \& Troì, A. (2006). A reassessment of the sections of the genus Cytisus Desf. (Cytiseae, Leguminosae). Taxon, 55(3), 733-746.

Danihelka, J., Chrtek, C., \& Kaplan, Z. (2012). Checklist of vascular plants of Czech Republic. Preslia, 84, 647-811.

Davis, P. H., Mill, R. R., \& Tan, K. (1988). Flora of Turkey and the East Aegean Islands. Vol. 10, Suppl. 1. Edinburgh.

Davydov, D. A., \& Gomlya, L. M. (2016). Anotovanyi spysok flory Rozhayivskoho botanichnoho zakaznyka ta pryleglykh do nioho stepovykh dilianok (Poltavska oblast) [An annotated checklist of the flora of "Rozhayivskiy" botanical reserve and adjacent steppe territories (Poltava region). Visnyk Problem Biolohii i Medytsyny, 4(1), 83-87 (in Ukrainian).

Didukh, Y. P. (Ed.). (2009). Chervona knyha Ukrayiny. Roslynnyi svit [The Red Data Book of Ukraine. Plant world]. Hlobalkonsaltynh, Kyiv (in Ukrainian).

Dimopoulos, P., Raus, T., Bergmeier, E., Constantinidis, T., Iatrou, G., Kokkini, S., Strid, A., \& Tzanoudakis, D. (2013). Vascular plants of Greece: An annotated checklist. Englera, 31, 1-372.

Dubovik, O. M. (1989). Novi vidomosti pro rid Rosa L. flory Ukrayiny [New data about the genus Rosa L. in the flora of Ukraine]. Ukrainian Botanical Journal, 46(3), 21-25 (in Ukrainian).

Dvirna, T. S. (2012). Znakhidky vydiv adventyvnykh roslyn na terytoriyi Romensko-Poltavskoho heobotanichnoho okruhu [Findings of alien plants on the territory of the Romny-Poltava geobotanical district]. Ukrainian Botanical Journal, 68(6), 847-852 (in Ukrainian).

Eyde, R. H. (1987). The case for keeping Cornus in the broad Linnaean sense. Systematic Botany, 12(4), 505-518

Fedoronchuk, M. M. (2007). Vydy sudynnykh roslyn, opysanykh z terytorii Ukrayiny, yikh typifikatsiya ta krytychnyi analiz: rodyna Apiaceae Lindl. (rody Sanicula L. - Trinia Hoffm.) [Species of vascular plants described from the territory of Ukraine, their typification and critical taxonomic analysis. The family Apiaceae Lindl. (genera Sanicula L. - Trinia Hoffm.]. Ukrainian Botanical Journal, 64(1), 13-21 (in Ukrainian).

Fedoronchuk, M. M. (2018). Konspekt rodyny Fabaceae u flori Ukrayiny. III. Pidrodyna Faboideae (tryba Fabeae) [A synopsis of the family Fabaceae in the flora of Ukraine. III. Subfamily Faboideae (tribe Fabeae)]. Ukrainian Botanical Journal, 75(5), 421-435 (in Ukrainian).

Fedoronchuk, M. M., \& Mosyakin, S. L. (2018). Konspekt rodyny Fabaceae u flori Ukrayiny. II. Pidrodyna Faboideae (tryby Galegeae, Hedysareae, Loteae, Cicereae) [A synopsis of the family Fabaceae in the flora of Ukraine. II. Subfamily Faboideae (tribes Galegeae, Hedysareae, Loteae and Cicereae)]. Ukrainian Botanical Journal, 75(4), 305-321 (in Ukrainian).

Foggi, B., Scholz, H., \& Valdés, B. (2005). The Euro+Med treatment of Festuca (Gramineae) - new names and new combinations in Festuca and allied genera. Willdenowia, 35(2), 241-244.

Freitag, H., \& Iamonico, D. (2015). Lectotypification of the name Polycnemum majus (Amaranthaceae s. str.). Phytotaxa, 202(3), 235-236.

Fuentes-Bazan, S., Uotila, P., \& Borsch, T. (2012). A novel phylogeny-based generic classification for Chenopodium sensu lato, and a tribal rearrangement of Chenopodioideae (Chenopodiaceae). Willdenowia, 42(1), 5-24. 
German, D. A. (2003). Zametki po rodu Alyssum L. (Cruciferae) Kazakhstana [Notes on the genus Alyssum L. (Cruciferae) in Kazakhstan]. Turczaninowia, 6(1), 45-57 (in Russian).

German, D. A. (2014). Revised typifications and nomenclatural notes in N Eurasian Cruciferae. Willdenowia, 44(3), 351-361.

German, D. A. (2015). Cruciferae (Brassicaceae): Alternative treatment for the "Conspectus of the vascular plants of Mongolia" (2014). Turczaninowia, 18(2), 39-67.

Gómez-Campo, C. (2002). The genus Guenthera Andr. in Bess. (Brassicaceae, Brassiceae). Anales del Jardin Botánico de Madrid, 60(2), 301-307.

Gomlya, L. M., \& Davydov, D. A. (2008). Flora vyshchykh sudynnykh roslyn Poltavskoho rayonu [Flora of the higher vascular plants of Poltava district]. FirmaTekhservis, Poltava (in Ukrainian).

Greuter, W. (2003a). The Euro+Med treatment of Astereae (Compositae) - generic concepts and required new names. Willdenowia, 33(1), 45-47.

Greuter, W. (2003b). The Euro+Med treatment of Cardueae (Compositae) - generic concepts and required new names. Willdenowia, 33(1), 49-61.

Greuter, W. (2003c). The Euro+Med treatment of Cichorieae (Compositae) generic concepts and required new names. Willdenowia, 33(2), 229-238.

Greuter, W. (2003d). The Euro+Med treatment of Senecioneae and the minor Compositae tribes - generic concepts and required new names, with an addendum to Cardueae. Willdenowia, 33(2), 245-250.

Greuter, W. (2009). Fabio Conti, Giovanna Abbate, Alessandro Alessandrini \& Carlo Blasi (ed.) - An annotated checklist of the Italian vascular flora. Optima Newsletter, 38, 36-37.

Greuter, W., Gutermann, W., \& Talavera, S. (2006). A preliminary conspectus of Scorzoneroides (Compositae, Cichorieae) with validation of the required new names. Willdenowia, 36(2), 689-692.

Greuter, W., Oberprieler, C., \& Vogt, R. (2003). The Euro+Med treatment of Anthemideae (Compositae) - generic concepts and required new names. Willdenowia, 33(1), 37-43.

Gutermann, W. (2009). Notulae nomenclaturales 29-40. Phyton (Horn), 49(1), $77-92$.

Gutiérrez-Larruscain, D., Santos-Vicente, M., Anderberg, A. A., Rico, E., \& Martínez-Ortega, M. M. (2018). Phylogeny of the Inula group (Asteraceae: Inuleae): Evidence from nuclear and plastid genomes and a recircumscription of Pentanema. Taxon, 67(1), 149-164.

Hernández-Ledesma, P., Berendsohn, W. G., Borsch, T., Mering, S., Akhani, H., Arias, S., Castañeda-Noa, I., Eggli, U., Eriksson, R., Flores-Olvera, H., FuentesBazán, S., Kadereit, G., Klak, C., Korotkova, N., Nyffeler, R., Ocampo, G., Ochoterena, H., Oxelman, B., Rabeler, R. K., Sanchez, A., Schlumpberger, B. O., \& Uotila, P. (2015). A taxonomic backbone for the global synthesis of species diversity in the angiosperm order Caryophyllales. Willdenowia, 45(3), 281-383.

Heywood, V. (2000). Floristics and monography - an uncertain future? Taxon, $50(2), 361-380$.

Holub, J. (1993). Leonurus intermedius, species nova - with additional notes on some other Leonurus taxa. Preslia, 65, 97-115.

Iamonico, D. (2017). Agrimonia eupatoria subsp. major stat. nov. (Rosaceae) and notes on the Agrimonia eupatoria aggregate. Phytotaxa, 298(2), 165-172.

Illichevskyi, S. O. (1927). Flora okolyts Poltavy (z povnym spyskom dykoyi roslynnosti) [Flora of the vicinities of Poltava town (with the full list of wild plants)]. Zapysky Poltavskoho Silskohospodarskoho Politekhnikuma, 1(2), 19-49 (in Ukrainian).

Illichevskyi, S. O. (1928). Roslynnist okolyts Poltavy [Vegetation of the vicinities of Poltava town]. Zapysky Poltavskoho Silskohospodarskoho Politekhnikuma, 2, 101-128 (in Ukrainian).

Jabbour, F., \& Renner, S. S. (2011). Consolida and Aconitella are an annual clade of Delphinium (Ranunculaceae) that diversified in the Mediterranean basin and the Irano-Turanian region. Taxon, 60(4), 1029-1040.

Jacobsen, N., \& von Bothmer, R. (1995). Taxonomy in the Hordeum murinum complex (Poaceae). Nordic Journal of Botany, 15(5), 449- 458.

Jafri, S. M. H. (1973). Flora of West Pakistan. Brassicaceae. University of Karachi, Karachi.

Jarvis, C. E. (1992). The Linnean Plant Name Typification Project. Botanical Journal of the Linnean Society, 109(4), 503-513.

Jarvis, C. E. (2007). Order out of Chaos. Linnean plant names and their types. The Linnean Society of London, London.

Jelenevskyi, A. G., \& Derviz-Sokolova, T. G. (1989). Liutiki (Ranunculus L., Ranunculaceae) Kavkaza [Buttercups (Ranunculus L., Ranunculaceae) of the Caucasus]. Bulletin of Moscow Society of Naturalists, Biological Series, 94(1), 112-122 (in Russian).

Kadereit, J. W., Albach, D. C., Ehrendorfer, F., Galbany-Casals, M., Garcia-Jacas, N., Gehrke, B., Kadereit, G., Kilian, N., Klein, J. T., Koch, M. A., Kropf, M., Oberprieler, C., Pirie, M. D., Ritz, C. M., Röser, M., Spalik, K., Susanna, A., Weigend, M., Welk, E., Wesche, K., Zhang, L.-B., \& Dillenberger, M. S. (2016). Which changes are needed to render all genera of the German flora monophyletic? Willdenowia, 46(1), 39-91.
Kamelin, R. V., \& Machmedov, A. M. (1990). Sistema roda Phlomoides (Lamiaceae) [The system of the genus Phlomoides (Lamiaceae)]. Botanicheskiy Zhurnal, 75(2), 241-250 (in Russian).

Kazmi, S. M. A. (1964). Revision der gattung Carduus (Compositae). Teil II. Mitteilungen der Botanischen Staatssammlung München, 5, 279-550.

Kotov, M. I. (1940). Opys novykh vydiv flory URSR. Descriptio specierum novarum ex Ucrainia. Journal Botanique de l'Academie des Sciences de la RSS d'Ukraine, 1(2), 275-279 (in Ukrainian).

Krasniak, O. I. (2012). Taksonomichni ta nomenklaturni problemy Bromus hordeaceus L. i B. mollis L. (Poaceae) [Taxonomic and nomenclatural problems of Bromus hordeaceus L. and B. mollis L. (Poaceae)]. Ukrainian Botanical Journal, 69(5), 688-693 (in Ukrainian).

Kurtto, A., Lampinen, R., Piirainen, M., \& Uotila, P. (2019). Checklist of the vascular plants of Finland. Finnish Museum of Natural History, Helsinki.

Lassen, P. (1989). A new delimitation of the genera Coronilla, Hippocrepis and Securigera (Fabaceae). Willdenowia, 19(1), 49-62.

Lazkov, G. A. (2006). Semeystvo hvozdichnye (Caryophyllaceae) vo flore Kyrgyzstana [The family Caryophyllaceae in the flora of Kyrgyzstan]. KMK Scientific Press, Moscow (in Russian).

Lesins, K. A., \& Lesins, I. (1979). Genus Medicago (Leguminosae): A taxogenetic study. Dr. W. Junk bv Publishers, The Hague-Boston-London.

Li, D. Z. (2008). Floristic and plant biogeography in China. Journal of Integrative Plant Biology, 50(7), 771-777.

Mathiesen, C., Scheen, A.-C., \& Lindqvist, C. (2011). Phylogeny and biogeography of the lamioid genus Phlomis (Lamiaceae). Kew Bulletin, 66(1), 81-99.

Melderis, A. (1978). Taxonomic notes on the tribe Triticeae (Gramineae), with special reference to the genera Elymus L. sensu lato, and Agropyron Gaertner sensu lato. Botanical Journal of the Linnean Society, 76(4), 369-384.

Melnikov, D. G. (2016). O taksonomicheskom statuse roda Acinos (Lamiaceae) [On the taxonomic status of the genus Acinos (Lamiaceae)]. Botanicheskiy Zhurnal, 101(1), 80-94 (in Russian).

Mennema, J. (1989). A taxonomic revision of Lamium (Lamiaceae). Leiden Botanical Series, 11, 1-198.

Meyer, F. K. (1973). Conspectus der "Thlaspl"-Arten Europas, Afrikas und Vorderasiens. Feddes Repertorium, 84(5-6), 449-470.

Mikheev, A. D. (2000). Obzor vidov Centaurea (Asteraceae) flory Kavkaza. 2. Podrody Lopholoma - Tetramorphaea [Synopsis of the species from the genus Centaurea (Asteraceae) in the flora of the Caucasus. 2. Subgenera LopholomaTetramorphaea]. Botanicheskiy Zhumal, 85(3), 116-124 (in Russian).

Moberg, R., \& Nilsson, O. (1991). Typification of Nordic vascular plants. 1. Names published by G. Wahlenberg. Nordic Journal of Botany, 11(3), 287-299.

Mosyakin, S. L. (2013). Nomenklaturno-taksonomichni zminy, shcho stosuyutsia deyakykh vydiv sudynnykh roslyn, zanesenykh do "Chervonoyi knyhy Ukrayiny" [Nomenclatural and taxonomic changes affecting the names of some vascular plants listed in the Red Data Book of Ukraine]. Ukrainian Botanical Journal, 70(2), 238-247 (in Ukrainian).

Mosyakin, S. L. (2017a). Notes on taxonomy and nomenclature of Chenopodium acerifolium and C. betaceum (C. strictum auct.) (Chenopodiaceae). Phytotaxa, 324(2), 139-154.

Mosyakin, S. L. (2017b). The first record of Salsola paulsenii (Chenopodiaceae) in Ukraine, with taxonomic and nomenclatural comments on related taxa. Ukrainian Botanical Journal, 74(5), 409-420.

Moysiyenko, I. I. (2008). Ogliad rodyny Limoniaceae Lincz. v Ukrayini [A review of the family Limoniaceae Lincz. in Ukraine]. Chornomorski Botanical Journal, 4(2), 161-174 (in Ukrainian).

Oberprieler, C., Himmelreich, S., \& Vogt, R. (2007). A new subtribal classification of the tribe Anthemideae (Compositae). Willdenowia, 37(1), 89-114

Oxelman, B., Lidén, M., Rabeler, R. K., \& Popp, M. (2001). A revised generic classification of the tribe Sileneae (Caryophyllaceae). Nordic Journal of Botany, 20(6), 743-748.

Pelser, P. B., Veldkamp, J.-F., \& van der Meijden, R. (2006). New combinations in Jacobaea Mill. (Asteraceae-Senecioneae). Compositae Newsletter, 44, 1-11.

Persson, K. (2007). Nomenclatural synopsis of the genus Colchicum (Colchicaceae), with some new species and combinations. Botanische Jahrbücher für Systematik, Pflanzengeschichte und Pflanzengeographie, 127(2), 165-242.

Peruzzi, L., Galasso, G., Domina, G., Bartolucci, F., Santangelo, A., Alessandrini, A., Astuti, G., D’Antraccoli, M., Roma-Marzio, F., Ardenghi, N. M. G., Barberis, G., Conti, F., Bernardo, L., Peccenini, S., Stinca, A., Wagensommer, R. P., Bonari, G., Iamonico, D., Iberite, M., Viciani, D., Del Guaccio, E., Guisso Del Galdo, G., Lastrucci, L., Villani, M., Brunu, A., Magrini, S., Pistarino, A., Brullo, S., Salmeri, C., Brundu, G., Clementi, M., carli, E., Vacca, G., Marcucci, R., Banfi, E., Longo, D., Di Pietro, R., \& Passalacqua, N. G. (2019). An inventory of the names of native, non-endemic vascular plants described from Italy, their loci classici and types. Phytotaxa, 410(1), 1-215.

Pesskova, I. M. (2004). Obzor roda Linaria Mill. (Scrophulariaceae) Vostochnoy Yevropy i Kavkaza [Review of the genus Linaria Mill. (Scrophulariaceae) of the Eastern Europe and the Caucasus]. Novitates Systematicae Plantarum Vascularium, 36, 182-208 (in Russian). 
Pimenov, M. G., \& Ostroumova, T. A. (2012). Zontichnye (Umbelliferae) Rossii [Umbelliferae of Russia]. KMK Scientific Press Ltd., Moscow (in Russian).

Pimenov, M. G., Kljuykov, E. V., \& Ostroumova, T. A. (2007). Critical taxonomic analysis of Dichoropetalum, Johrenia, Zeravschania and related genera of Umbelliferae-Apioideae-Peucedaneae. Willdenowia, 37(2), 465-502.

Polatschek, A. (2010). Revision der Gattung Erysimum (Cruciferae): Teil 1: Russland, die Nachfolgestaaten der USSR (excl. Georgien, Armenien, Azerbaidzan), China, Indien, Pakistan, Japan und Korea. Annalen des Naturhistorischen Museums in Wien, serie B, 111, 181-275.

Pruski, J. F. (2005). Nomenclatural notes on Cyclachaena (Compositae: Heliantheae), resurrection and lectotypification of $I v a$ sect. Pycrotus, and report of $I v a$ (syn. Cyclachaena) as new to Armeria. Compositae Newsletter, 42, 32-42.

Rauschert, S. (1973). Zur Nomenklatur der Farn- und Blutenpflanzen Deutschlands (III). Feddes Repertorium, 83(9-10), 645-662.

Saarela, J. M., Peterson, P. M., Keane, R. M., Cayouette, J., \& Graham, S. W. (2007). Molecular phylogenetics of Bromus (Poaceae: Pooideae) based on chloroplast and nuclear DNA sequence data. Aliso, 23(1), 379-396.

Schäfer, H., Hechenleitner, P., Santos-Guerra, A., Menezes de Sequeira, M., Pennington, R. T., Kenicer, G., \& Carine, M. A. (2012). Systematics, biogeography and character evolution of the legume tribe Fabeae with special focus on the middle-Atlantic island lineages. BMC Evolutionary Biology, 12, 1-250.

Schlee, M., Göker, M., Grimm, G. W., \& Hemleben, V. (2011). Genetic patterns in the Lathyrus pannonicus complex (Fabaceae) reflect ecological differentiation rather than biogeography and traditional subspecific division. Botanical Journal of the Linnean Society, 165(4), 402-421.

Sennikov, A. N., \& Kurtto, A. (2019). The taxonomy and invasion status assessment of Erigeron annuus s.l. (Asteraceae) in East Fennoscandia. Memoranda Societatis pro Fauna et Flora Fennica, 95, 40-59.

Sennikov, A. N., Tojibaev, K. S., Khassanov, F. O., \& Beshko, N. Y. (2016). The flora of Uzbekistan project. Phytotaxa, 282(2), 107-118.

Shaparenko, I. (2012). Novi znakhidky ridkisnykh stepovykh vydiv roslyn u baseyni richky Poluzirya [New findings of rare steppe plant species in the basin of Poluzirya river]. Visnyk Lvivskoho Universytetu, Seriya Biolohichna, 58, 97-106 (in Ukrainian).

Shi, S., Li, J., Sun, J., Yu, J., \& Zhou, S. (2013). Phylogeny and classification of Prunus sensu lato (Rosaceae). Journal of Integrative Plant Biology, 55(11), 1069-1079.

Smejkal, M. (1971). Revision der tschechoslowakischen arten der gattung Camelina Crantz (Cruciferae). Preslia, 43, 318-337.
Soják, J. (2004). Potentilla L. (Rosaceae) and related genera in the former USSR (identification key, checklist and figures). Notes on Potentilla XVI. Botanische Jahrbücher für Systematik, Pflanzengeschichte und Pflanzengeographie, 125(3), 253-340.

Soják, J. (2010). Argentina Hill, a genus distinct from Potentilla (Rosaceae). Thaiszia - Journal of Botany, 20, 91-97.

Sonboli, A., Stroka, K., Osaloo, S. K., \& Oberprieler, C. (2012). Molecular phylogeny and taxonomy of Tanacetum L. (Compositae, Anthemideae) inferred from nrDNA ITS and cpDNA trnH-psbA sequence variation. Plant Systematic and Evolution, 298, 431-444.

Soreng, R. J., Peterson, P. M., Romaschenko, K., Davidse, G., Teisher, J. K., Clark, L. G., Barberá, P., Gillespie, L. J., \& Zuloaga, F. O. (2017). A worlwide phylogenetic classification of the Poaceae (Gramineae) II: An update and a comparison of two 2015 classifications. Journal of Systematics and Evolution, 55(4), 259-290.

Styles, B. T. (1962). The taxonomy of Polygonum aviculare and its allies in Britain. Watsonia, 5(4), 177-214.

Suchorukow, A. P. (2007). Zur systematik und chorologie der in Russland und den benachbarten staaten (in den grenzen der ehemaligen USSR) vorkommenden Atriplex-arten (Chenopodiaceae). Annalen des Naturhistorischen Museums in Wien, Series B, 108, 307-420.

Turland, N. J., Wiersema, J. H., Barrie, F. R., Greuter, W., Hawksworth, D. L., Herendeen, P. S., Knapp, S., Kusber, W.-H., Li, D.-Z., Marhold, K., May, T. W., McNeill, J., Monro, A. M., Prado, J., Price, M. J., \& Smith, G. F. (2018). International Code of Nomenclature for algae, fungi, and plants (Shenzhen Code) adopted by the Nineteenth International Botanical Congress Shenzhen, China July 2017. Regnum Vegetabile 159. Koeltz Botanical Books, Glashütten.

Tzvelev, N. N. (2011). O nekotorykh vidakh ovsianits (Festuca L., Poaceae) Rossii [On the some species of the genus Festuca L. (Poaceae) of Russia]. Botanika (Minsk), 40, 114-130 (in Russian).

Verloove, F., \& Lambinon, J. (2014). The sixth edition of the Nouvelle Flore de la Belgique: Nomenclatural and taxonomic remarks. Dumortiera, 104, 7-40.

Wrigley, F. (1986). Taxonomy and chorology of Silene section Otites (Caryophyllaceae). Annales Botanici Fennici, 23, 69-81.

Zhygalova, S. L. (2016). Rodyny Ulmaceae Mirb. ta Celtidaceae Endl. u flori Ukrayiny [The family Ulmaceae Mirb. and Celtidaceae Endl. in the flora of Ukraine]. Plant Introduction, 72, 52-58 (in Ukrainian). 